\title{
The non-anticommutative supersymmetric Wess-Zumino model
}

\author{
I. Jack, ${ }^{a}$ D.R.T. Jones ${ }^{b}$ and R. Purdy ${ }^{a}$ \\ ${ }^{a}$ Dept. of Mathematical Sciences, University of Liverpool, \\ Liverpool L69 3BX, U.K. \\ ${ }^{b}$ TH Division, CERN, \\ 1211 Geneva 23, Switzerland* \\ E-mail: dij@liverpool.ac.uk, drtj@liverpool.ac.ul, \\ robert.purdy@liverpool.ac.uk
}

\begin{abstract}
We discuss the non-anticommutative $\left(\mathcal{N}=\frac{1}{2}\right)$ supersymmetric Wess-Zumino model in four dimensions. Firstly we introduce differential operators which implement the non-anticommutative supersymmetry algebra acting on the component fields and action. Then we perform the renormalisation of the model up to two-loop order, including the complete set of terms necessary for renormalisability. We show that (at least up to this order) the results obtained when we eliminate the auxiliary field after renormalisation are equivalent to those obtained when we eliminate the auxiliary fields before quantisation.
\end{abstract}

KEYwords: Superspaces, Renormalization Group.

${ }^{*}$ Address Oct 1st-Dec 31st 2008 


\section{Contents}

1. Introduction 1

2. Representation of the supersymmetry algebra; the undeformed case 2

3. Representation of the supersymmetry algebra; the deformed case 3

4. Renormalisation 7

5. Conclusions 26

\section{Introduction}

The subject of deformed quantum field theories has attracted renewed attention in recent years due to their natural appearance in string theory. Initial studies were devoted to theories on non-commutative spacetime in which the commutator of the spacetime coordinates becomes non-zero. More recently [1]- 9], non-anticommutative supersymmetric theories have been constructed by deforming the anticommutators of the grassmann coordinates $\theta^{\alpha}$ (while leaving the anticommutators of the $\bar{\theta}^{\dot{\alpha}}$ unaltered). Consequently, the anticommutators of the supersymmetry generators $\bar{Q}_{\dot{\alpha}}$ are deformed while those of the $Q_{\alpha}$ are unchanged. Non-anticommutative versions of the Wess-Zumino model and supersymmetric gauge theories have been formulated in four dimensions [10, 11] and their renormalisability discussed [12]-16], with explicit computations up to two loops [17] for the Wess-Zumino model and one loop for gauge theories [18]-22].

More recently still, non-anticommutative theories in two dimensions have been considered. On the one hand, non-anticommutative versions of particular non-linear $\sigma$-models have been constructed (by dimensional reduction from four dimensions) [23] and the oneloop corrections computed [24]; on the other hand, a non-anticommutative version of the general $\mathcal{N}=2$ Kähler $\sigma$-model has been constructed directly in two dimensions, initially in refs. [25, 26] but then given an elegant reformulation in refs. [27, 28]. The one-loop divergences for this model were computed in ref. [29], where it was found convenient to introduce differential operators implementing the deformed supersymmetry algebra acting on the component fields.

In this article we return to a closer examination of the non-anticommutative WessZumino model in four dimensions. Firstly, we show how analogues of the differential operators introduced in ref. [29] may easily be constructed to implement the nonanticommutative supersymmetry algebra for this model in its component formulation. Next we re-examine the two-loop calculation first performed in ref. [17], showing that to correctly 
obtain results for the theory where the auxiliary fields have been eliminated from those for the uneliminated theory, it is necessary to include in the classical action separate couplings for all the terms which may be generated by the renormalisation process. We show that (at least up to two-loop order) the results obtained when we eliminate the auxiliary field after renormalisation are equivalent to those obtained when we eliminate the auxiliary fields before quantisation.

\section{Representation of the supersymmetry algebra; the undeformed case}

We follow the analysis of ref. [29] in determining differential operators which represent the supersymmetry algebra in the undeformed and deformed cases. The supersymmetry charges are

$$
Q_{\alpha}=\frac{\partial}{\partial \theta^{\alpha}}, \quad \bar{Q}_{\dot{\alpha}}=-\frac{\partial}{\partial \bar{\theta}^{\dot{\alpha}}}+2 i \theta^{\alpha} \partial_{\alpha \dot{\alpha}}
$$

where

$$
\begin{aligned}
\partial_{\alpha \dot{\alpha}} & =\sigma_{\alpha \dot{\alpha}}^{\mu} \frac{\partial}{\partial y^{\mu}}, \\
y^{\mu} & =x^{\mu}+i \theta^{\alpha} \sigma_{\alpha \dot{\alpha}}^{\mu} \bar{\theta}^{\dot{\alpha}} .
\end{aligned}
$$

They satisfy the algebra

$$
\begin{aligned}
& \left\{Q_{\alpha}, Q_{\beta}\right\}=0, \quad\left\{\bar{Q}_{\dot{\alpha}}, \bar{Q}_{\dot{\beta}}\right\}=0, \\
& \left\{\bar{Q}_{\dot{\alpha}}, Q_{\alpha}\right\}=2 i \partial_{\alpha \dot{\alpha}} .
\end{aligned}
$$

The superfields have expansions in terms of component fields given by

$$
\begin{aligned}
& \Phi=\phi+\sqrt{2} \theta \psi+\theta^{2} F, \\
& \bar{\Phi}=\bar{\phi}+\sqrt{2} \overline{\theta \psi}-2 i \theta \sigma^{\mu} \bar{\theta} \partial_{\mu} \bar{\phi}+\bar{\theta}^{2}\left[\bar{F}+i \sqrt{2} \theta \partial \bar{\psi}+\theta^{2} \partial^{2} \bar{\phi}\right],
\end{aligned}
$$

where the component fields are functions of $y^{\mu}$, as defined in eq. (2.2). It is useful to represent the charges $Q_{\alpha}, \bar{Q}_{\dot{\alpha}}$ by differential operators $q_{\alpha}, \bar{q}_{\dot{\alpha}}^{0}$ acting on the fields, i.e.

$$
\begin{aligned}
{\left[Q_{\alpha}, \Phi\right] } & =q_{\alpha} \Phi \\
{\left[\bar{Q}_{\dot{\alpha}}, \Phi\right] } & =\bar{q}_{\dot{\alpha}}^{0} \Phi
\end{aligned}
$$

(with similar expressions for $\bar{\Phi}$ ) where

$$
\begin{aligned}
& \frac{1}{\sqrt{2}} q_{\alpha}=\psi_{\alpha} \frac{\partial}{\partial \phi}+F \frac{\partial}{\partial \psi^{\alpha}}-i\left[\partial_{\alpha \dot{\alpha}} \bar{\phi}\right] \frac{\partial}{\partial \bar{\psi}_{\dot{\alpha}}}+i\left[\partial_{\alpha \dot{\alpha}} \bar{\psi}^{\dot{\alpha}}\right] \frac{\partial}{\partial \bar{F}}, \\
& \frac{1}{\sqrt{2}} \bar{q}_{\dot{\alpha}}^{0}=\bar{\psi}_{\dot{\alpha}} \frac{\partial}{\partial \bar{\phi}}-\bar{F} \frac{\partial}{\partial \bar{\psi}^{\dot{\alpha}}}-i\left[\partial_{\alpha \dot{\alpha}} \phi\right] \frac{\partial}{\partial \psi_{\alpha}}-i\left[\partial_{\alpha \dot{\alpha}} \psi^{\alpha}\right] \frac{\partial}{\partial F} .
\end{aligned}
$$

The superscript " 0 " is in anticipation of a different form for $\bar{q}_{\dot{\alpha}}$ in the deformed case; while $q_{\alpha}$, on the other hand, will be unchanged. Our convention will be that a derivative or other operator acts on everything to its right, unless enclosed in square brackets. (By the way, note that

$$
\left.\epsilon_{\alpha \beta} \frac{\partial}{\partial \psi_{\beta}}=-\frac{\partial}{\partial \psi^{\alpha}} .\right)
$$




\section{Representation of the supersymmetry algebra; the deformed case}

In this section we repeat the analysis of the previous section for the case of deformed supersymmetry. For the deformed version we take

$$
\left\{\bar{\theta}^{\dot{\alpha}}, \bar{\theta}^{\dot{\beta}}\right\}=0, \quad\left\{\theta^{\alpha}, \theta^{\beta}\right\}=C^{\alpha \beta} .
$$

The charges then satisfy the algebra

$$
\begin{aligned}
\left\{Q_{\alpha}, Q_{\beta}\right\} & =0, \quad\left\{\bar{Q}_{\dot{\alpha}}, \bar{Q}_{\dot{\beta}}\right\}=-4 C^{\alpha \beta} \partial_{\alpha \dot{\alpha}} \partial_{\beta \dot{\beta}}, \\
\left\{\bar{Q}_{\dot{\alpha}}, Q_{\alpha}\right\} & =2 i \partial_{\alpha \dot{\alpha}} .
\end{aligned}
$$

The non-anticommutativity is implemented at the level of superfields by introducing the Moyal *-product, which satisfies

$$
\begin{aligned}
& \theta^{\alpha} * \theta^{\beta}=-\frac{1}{2} \epsilon^{\alpha \beta} \theta^{2}+\frac{1}{2} C^{\alpha \beta}, \\
& \theta^{\alpha} * \theta^{2}=C^{\alpha \beta} \theta_{\beta} \\
& \theta^{2} * \theta^{2}=-\operatorname{det} C \equiv \frac{1}{M^{2}} .
\end{aligned}
$$

We now wish to construct differential operators $\bar{q}_{\dot{\alpha}}$ representing the effects of $\bar{Q}_{\dot{\alpha}}$ in the deformed case in a similar manner to eq. (2.6), extending $\bar{q}_{\dot{\alpha}}^{0}$ given in eq. (2.7b) for the undeformed case. (As mentioned before, the operators $q_{\alpha}$ are unchanged by the deformation.) We start by examining the effects of $\bar{Q}_{\dot{\alpha}}$ on powers of $\Phi$ alone, since mixed products of $\Phi$ and $\bar{\Phi}$ present additional complications. Defining

$$
I_{r}^{(n)}(\phi, F)=\int_{-\frac{1}{2}}^{\frac{1}{2}} d \xi\left(\frac{\xi}{2 M}\right)^{r}\left(\phi+\frac{2 \xi}{M} F\right)^{n}
$$

it is straightforward to show using the methods of ref. [27] that

$$
\Phi_{*}^{n}=\left(1+\theta q-\frac{1}{4} \theta^{2} q^{2}\right)\left(I_{0}^{(n)}-q^{2} I_{1}^{(n)}\right),
$$

where $\Phi_{*}^{n}$ denotes the $*$-product of $n \Phi$ 's. Then acting on $\Phi_{*}^{n}, \bar{Q}_{\dot{\alpha}}$ is represented by

$$
\bar{q}_{\dot{\alpha}}^{\Phi}=\bar{q}_{\dot{\alpha}}^{0}-i(q C \partial)_{\dot{\alpha}}+4 i\left(-q^{2}\left[\partial_{\alpha \dot{\alpha}} q^{\alpha}\right] \widetilde{\mathcal{O}}+\partial_{\alpha \dot{\alpha}} q^{\alpha} \mathcal{O}+\left[\partial_{\alpha \dot{\alpha}} q^{\alpha}\right] \mathcal{O}\right)
$$

Here

$$
\begin{aligned}
& \mathcal{O} I_{0}^{(n)}=I_{1}^{(n)}, \\
& \mathcal{O} I_{1}^{(n)}=I_{2}^{(n)}-\widetilde{\mathcal{O}} I_{0}^{(n)} .
\end{aligned}
$$

These properties are guaranteed by the following definitions:

$$
\begin{aligned}
\mathcal{O} & =\sum_{r=1}^{\infty} a_{r}\left(\frac{1}{4 M^{2}}\right)^{r}\left(4 F \frac{\partial}{\partial \phi}\right)^{2 r-1}, \\
\widetilde{\mathcal{O}} & =\sum_{r=1}^{\infty}(2 r-1) a_{r}\left(\frac{1}{4 M^{2}}\right)^{r}\left(4 F \frac{\partial}{\partial \phi}\right)^{2 r-2},
\end{aligned}
$$


where the $a_{r}$ must satisfy for each $n \geq 1$ [29]

$$
\sum_{r=0}^{n-1} \frac{a_{n-r}}{2^{2 r}(2 r+1)(2 r) !}=\frac{1}{2^{2 n}(2 n+1)(2 n-1) !},
$$

the first few being given by

$$
a_{1}=\frac{1}{12}, \quad a_{2}=-\frac{1}{720}, \quad a_{3}=\frac{1}{2^{5} \cdot 3^{3} \cdot 5 \cdot 7} .
$$

To check that the operators in eq. (3.6) do indeed represent the operators $\bar{Q}_{\dot{\alpha}}$ according to

$$
\left[\bar{Q}_{\dot{\alpha}}, \Phi_{*}^{n}\right]_{*}=\bar{q}_{\dot{\alpha}}^{\Phi} \Phi_{*}^{n}
$$

(where $[,]_{*}$ represents the commutator evaluated using $*$-products) we need to use eqs. (3.7) in conjunction with

$$
\bar{q}_{\dot{\alpha}}^{0} I_{r}^{(n)}=-i\left[\partial_{\alpha \dot{\alpha}} q^{\alpha}\right] I_{r+1}^{(n)} .
$$

Of course it is not sufficient to reproduce the effects of $\bar{Q}_{\dot{\alpha}}$ on $*$-products of $\Phi$ alone; we saw in the two-dimensional case that it was necessary to consider the effect on deformed versions of general polynomials in $\Phi$ and $\bar{\Phi}$, such as the Kähler potential. In the case of the four-dimensional Wess-Zumino model, to investigate the divergence structure it would be sufficient to consider only the effects of $\bar{Q}_{\dot{\alpha}}$ on cubic superpotentials in either $\Phi$ or $\bar{\Phi}$, or on $\Phi * \bar{\Phi}$. However, in general, if one were interested in contributions to the effective action, one would need once again to consider deformed versions of general polynomials; and so we shall again take the Kähler potential as an example.

For an undeformed Kähler potential

$$
K[\Phi, \bar{\Phi}]=\sum_{n, m} K_{n, m} \Phi^{n} \bar{\Phi}^{m}
$$

the natural definition of the deformed Kähler potential is

$$
K_{*}[\Phi, \bar{\Phi}]=\sum_{n, m} K_{n, m}\left[\Phi^{n} \bar{\Phi}^{m}\right]_{*}
$$

where $\left[\Phi^{n} \bar{\Phi}^{m}\right]_{*}$ represents the symmetrised *-product of $n \Phi$ 's and $m \bar{\Phi}$ 's. It can be shown that

$$
\begin{aligned}
K_{*}[\Phi, \bar{\Phi}]= & \left(1+\theta q-\frac{1}{4} \theta^{2} q^{2}\right)\left[1+\bar{\theta} \bar{q}^{0 \prime \prime}-\frac{1}{4} \bar{\theta}^{2}\left(\bar{q}^{0 \prime \prime}\right)^{2}\right] \\
& {\left[K_{0}(\phi, F, \bar{\phi})-q^{2} K_{1}(\phi, F, \bar{\phi})\right] } \\
& -\frac{1}{4 M^{2}} \bar{\theta}^{2} q^{\prime 2}\left(\partial^{\prime \prime}\right)^{2} K_{0}(\phi, F, \bar{\phi}),
\end{aligned}
$$

where

$$
K_{m}(\phi, F, \bar{\phi})=\int_{-\frac{1}{2}}^{\frac{1}{2}} d \xi\left(\frac{\xi}{2 M}\right)^{m} K\left(\phi+\frac{2 \xi}{M} F, \bar{\phi}\right)
$$


Here a prime denotes the part of the operator containing derivatives with respect to the chiral (but not the anti-chiral) fields, and correspondingly a double prime denotes the part of the operator containing derivatives with respect to the anti-chiral (but not the chiral) fields. Moreover,

$$
\partial_{\mu}^{\prime \prime}=\left[\partial_{\mu} \bar{\phi}\right] \frac{\partial}{\partial \bar{\phi}}+\left[\partial_{\mu} \bar{\psi}_{\dot{\alpha}}\right] \frac{\partial}{\partial \bar{\psi}_{\dot{\alpha}}}+\left[\partial_{\mu} \bar{F}\right] \frac{\partial}{\partial \bar{F}},
$$

with a similar expression for $\partial_{\mu}^{\prime}$. The corresponding version of the operator representing $\bar{Q}_{\dot{\alpha}}$ is $\bar{q}_{\dot{\alpha}}$ defined by

$$
\begin{aligned}
\bar{q}_{\dot{\alpha}}= & \bar{q}_{\dot{\alpha}}^{0}-i(q C \partial)_{\dot{\alpha}}-\frac{i}{4 M^{2}}\left(\partial_{\alpha \dot{\alpha}}^{\prime \prime} q^{\prime \prime \alpha} q^{\prime 2}+\partial_{\alpha \dot{\alpha}}^{\prime} q^{\prime \alpha} q^{\prime \prime 2}\right) \\
& +4 i\left(-q^{\prime 2}\left[\partial_{\alpha \dot{\alpha}}^{\prime} q^{\prime \alpha}\right] \widetilde{\mathcal{O}}+\partial_{\alpha \dot{\alpha}}^{\prime} q^{\prime \alpha} \mathcal{O}+\left[\partial_{\alpha \dot{\alpha}}^{\prime} q^{\prime \alpha}\right] \mathcal{O}\right)
\end{aligned}
$$

We can verify that these operators do indeed implement the operators $\bar{Q}_{\dot{\alpha}}$ according to

$$
\left[\bar{Q}_{\dot{\alpha}}, K_{*}\right]_{*}=\bar{q}_{\dot{\alpha}} K_{*}
$$

using the analogue of eq. (3.7) for the Kähler potential,

$$
\begin{aligned}
& \mathcal{O} K_{0}=K_{1}, \\
& \mathcal{O} K_{1}=K_{2}-\widetilde{\mathcal{O}} K_{0}
\end{aligned}
$$

together with the analogue of eq. (3.12),

$$
\bar{q}_{\dot{\alpha}}^{\prime 0} K_{r}=-i\left[\partial_{\alpha \dot{\alpha}}^{\prime} q^{\prime \alpha}\right] K_{r+1}, \quad q_{\alpha}^{\prime \prime} K_{0}=q_{\alpha}^{\prime \prime} K_{1}=0 .
$$

Moreover, it is easy to check that the operators $q_{\alpha}$ in eq. (2.7a) and $\bar{q}_{\dot{\alpha}}$ in eq. (3.18) satisfy the anticommutation relations of eq. (3.2), using

$$
\left[\bar{q}_{\dot{\alpha}}^{0}, F \frac{\partial}{\partial \phi}\right]=-i\left[\partial_{\alpha \dot{\alpha}}^{\prime} q^{\prime \alpha}\right]
$$

(which implies

$$
\left.\left[\bar{q}_{\dot{\alpha}}^{0}, \mathcal{O}\right]=-i\left[\partial_{\alpha \dot{\alpha}}^{\prime} q^{\prime \alpha}\right] \widetilde{\mathcal{O}}\right)
$$

The kinetic part of the standard Wess-Zumino model may be obtained as in ref. 29] from eq. (3.15) taking simply $K[\Phi, \bar{\Phi}]=\Phi \bar{\Phi}$,

$$
\begin{aligned}
S_{\text {kin }} & =\int d^{4} x d^{2} \theta d^{2} \bar{\theta} \Phi * \bar{\Phi}=\frac{1}{16} \int d^{4} x q^{2} \bar{q}^{2} \phi \bar{\phi} \\
& =\int d^{4} x\left(\partial^{\mu} \bar{\phi} \partial_{\mu} \phi+i \bar{\psi} \bar{\sigma}^{\mu} \partial_{\mu} \psi+\bar{F} F\right),
\end{aligned}
$$

so we see that the kinetic terms are undeformed. We see from eq. (3.5) that the holomorphic potential terms are given by

$$
S_{W}=-\int d^{4} x \int d^{2} \theta\left[\frac{1}{2} m \Phi_{*}^{2}+\frac{1}{6} y \Phi_{*}^{3}\right]=\frac{1}{4} \int d^{4} x q^{2}\left[\frac{1}{2} m I_{0}^{(2)}+\frac{1}{6} y I_{0}^{(3)}\right]
$$


which leads to

$$
S_{W}=\int d^{4} x\left[\frac{1}{2} m\left(\psi^{2}-F \phi\right)+\frac{1}{2} y\left(\phi \psi^{2}-F \phi^{2}\right)+\frac{1}{6} y(\operatorname{det} C) F^{3}\right] .
$$

Since $\bar{\Phi}_{*}^{n}=\bar{\Phi}^{n}$, the antiholomorphic potential terms are given by

$$
S_{\bar{W}}=-\int d^{4} x \int d^{2} \bar{\theta}\left[\frac{1}{2} \bar{m} \bar{\Phi}^{2}+\frac{1}{6} \bar{y} \bar{\Phi}^{3}\right]=\frac{1}{4} \int d^{4} x \bar{q}^{2}\left[\frac{1}{2} \bar{m} \bar{\phi}^{2}+\frac{1}{6} \bar{y} \bar{\phi}^{3}\right]
$$

which leads to

$$
S_{\bar{W}}=\int d^{4} x\left[\frac{1}{2} \bar{m}\left(\bar{\psi}^{2}-\overline{F \phi}\right)+\frac{1}{2} \bar{y}\left(\overline{\phi \psi}^{2}-\overline{F \phi}^{2}\right)\right]
$$

with no deformation (the deformed part of $\bar{q}$ in eq. (3.18) having no effect on a function of $\bar{\phi})$. The full classical $\mathcal{N}=\frac{1}{2}$ action is therefore

$$
\begin{aligned}
S= & \int d^{4} x\left(\partial^{\mu} \bar{\phi} \partial_{\mu} \phi+i \bar{\psi} \bar{\sigma}^{\mu} \partial_{\mu} \psi+\bar{F} F-G F-\overline{G F}\right. \\
& \left.+\frac{1}{2} y \phi \psi^{2}+\frac{1}{2} \bar{y}_{\phi \psi}^{2}+\frac{1}{2} m \psi^{2}+\frac{1}{2} \bar{m} \bar{\psi}^{2}+\frac{1}{6} y(\operatorname{det} C) F^{3}\right),
\end{aligned}
$$

where $G=m \phi+\frac{1}{2} y \phi^{2}$. This action was first derived in ref. [10] by taking the standard undeformed $(\mathcal{N}=1)$ action in superfields and replacing ordinary products by Moyal *-products.

In the undeformed case, expressions like those in eqs. (3.24), (3.25) and (3.27) in terms of $q_{\alpha}$ and $\bar{q}_{\dot{\alpha}}^{0}$ encapsulate the supersymmetry of the undeformed action $S_{0}$ due to the nilpotency of $q_{\alpha}, \bar{q}_{\dot{\alpha}}$ and the fact that $q_{\alpha}, \bar{q}_{\dot{\alpha}}^{0}$ annihilate functions of $\bar{\phi}, \phi$, respectively, leading to

$$
q_{\alpha} S_{0}=\bar{q}_{\dot{\alpha}}^{0} S_{0}=0
$$

In the deformed case, although

$$
q_{\alpha} S_{\text {kin }}=\bar{q}_{\dot{\alpha}} S_{\text {kin }}=q_{\alpha} S_{W}=q_{\alpha} S_{\bar{W}}=\bar{q}_{\dot{\alpha}} S_{\bar{W}}=0,
$$

it is not the case that $\bar{q}_{\dot{\alpha}} S_{W}=0$; indeed it is no longer the case that $\bar{q}_{\dot{\alpha}}^{0} S_{W}=0$ either. It is only the tranformations generated by $Q_{\alpha}$ that are a symmetry of the deformed action with potential. It is worth mentioning that in the two-dimensional case (where we considered only the kinetic part of the action derived from the Kähler potential), despite our classical action being annihilated by $q$ and $\bar{q}$, the divergent quantum corrections were only annihilated by $q$. In fact it is only for simple linear field transformations that one can prove that an invariance of the classical action results in a similar invariance of the quantum corrections; for non-linear gauge transformations the quantum invariance is encapsulated in Ward identities, and for transformations such as our deformed $\bar{q}_{\dot{\alpha}}$ some other formulation may be possible. 


\section{Renormalisation}

In this section we discuss the renormalisation of the non-anticommutative Wess-Zumino model up to two loops. Two-loop calculations for this model were first performed by Grisaru et al [17]; here we extend their calculation by including from the outset the full set of terms which can be generated by renormalisation. We shall postpone until later a detailed comparison of our results with theirs.

The only effect of the non-anticommutativity in the component action of eq. (3.29) is the final $(\operatorname{det} C)$ term. As we emphasised at the end of the last section, the deformed action is only invariant under the transformations generated by $Q_{\alpha}$. This term is in fact separately invariant under these residual $\mathcal{N}=\frac{1}{2}$ transformations and so there is no reason for the coefficient to evolve in the same manner as the Yukawa coupling in the $\mathcal{N}=1$ part of the action; and therefore we are at liberty (possibly even obliged) to introduce this term with its own separate coefficient. In fact, this term generates one-loop divergences whose cancellation requires $(\operatorname{det} C) F^{2} \bar{G}$ and $\bar{m}^{2}(\operatorname{det} C) F^{2}$ terms in the action; and these terms in turn generate further $(\operatorname{det} C) F^{3}$ divergences together with other new terms. All these terms should be included in the classical action with their own coefficients in order to guarantee renormalisability. It is easy to see which additional terms can be generated [16]. (For a complete analysis in the general, gauged case, see ref. [22].) The action has a "pseudo R-symmetry" under

$$
\phi \rightarrow e^{-i \omega} \phi, \quad F \rightarrow e^{i \omega} F, \quad C^{\alpha \beta} \rightarrow e^{-2 i \omega} C^{\alpha \beta}, \quad y \rightarrow e^{i \omega} y,
$$

$\bar{F}, \bar{\phi}$ and $\bar{y}$ transforming with opposite charges to $F, \phi$ and $y$ respectively, and $\psi, \bar{\psi}$ being neutral; and also a "pseudo-chiral symmetry" under

$$
\phi \rightarrow e^{i \gamma} \phi, \quad m \rightarrow e^{-2 i \gamma} m, \quad y \rightarrow e^{-3 i \gamma} y
$$

$F$ and $\psi$ transforming in a similar fashion to $\phi$ and barred quantities transforming with opposite charges. The divergent terms which can arise subject to these invariances have been enumerated [16] and consist (for the ungauged case) of

$$
\begin{array}{rll}
\bar{y}^{-1}(\operatorname{det} C) F^{3}, & \bar{y}^{-1}(\operatorname{det} C) F^{2} \bar{G}, & \bar{y}^{-1}(\operatorname{det} C) F \bar{G}^{2}, \\
\bar{y}^{-1}(\operatorname{det} C) \bar{G}^{3}, & \bar{y}^{-2} \bar{m}^{2}(\operatorname{det} C) F^{2}, & \bar{y}^{-2} \bar{m}^{2}(\operatorname{det} C) F \bar{G}, \\
\bar{y}^{-2} \bar{m}^{2}(\operatorname{det} C) \bar{G}^{2}, & \bar{y}^{-3} \bar{m}^{4}(\operatorname{det} C) F, & \bar{y}^{-3} \bar{m}^{4}(\operatorname{det} C) \bar{G} .
\end{array}
$$

We have anticipated here the fact, adumbrated in ref. [17] and proved in ref. [14, that the divergences form combinations of $F$ and $\bar{G}$; so that we need only a single coupling to remove divergences in (for instance) $(\operatorname{det} C) F^{2} \bar{y} \bar{\phi}^{2}$ and $(\operatorname{det} C) F^{2} \bar{m} \bar{\phi}$. We have included in (4.3) the appropriate factors of $\bar{y}$ for invariance under the pseudo-chiral symmetry. These factors are not uniquely determined since $y \bar{y}$ is invariant under this symmetry; the choice we have made is both concise and motivated by later considerations. Each of these terms is separately $\mathcal{N}=\frac{1}{2}$ invariant and so there is nothing in the classical theory to determine their coefficients; but we shall investigate whether renormalisability has anything to tell us about their values. In fact, some of the terms listed in (4.3) could be omitted and still leave 
a renormalisable theory (at least up to two-loop order); but nevertheless for completeness we shall include all the above terms in our classical action. (As pointed out in ref. [22], terms of the form $y F \psi C \psi$ and $\bar{\phi}^{2} \psi C \psi$ are possible; but not in the present ungauged case with only one chiral field.)

We are therefore led to the action

$$
\begin{aligned}
S= & \int d^{4} x\left(\partial^{\mu} \bar{\phi} \partial_{\mu} \phi+i \bar{\psi} \bar{\sigma}^{\mu} \partial_{\mu} \psi+\bar{F} F-G F-\overline{G F}\right. \\
& +\frac{1}{2} y \phi \psi^{2}+\frac{1}{2} \bar{y}_{\phi \psi}^{2}+\frac{1}{2} m \psi^{2}+\frac{1}{2} \bar{m} \bar{\psi}^{2} \\
& +\bar{y}^{-1}\left[\frac{1}{6} k_{1} F^{3}+\frac{1}{2} k_{2} F^{2} \bar{G}+\frac{1}{2} k_{3} \bar{G}^{2}+\frac{1}{6} k_{4} \bar{G}^{3}\right] \\
& \left.+\frac{1}{2} \bar{y}^{-2} \bar{m}^{2}\left[k_{5} F^{2}+2 k_{6} F \bar{G}+k_{7} \bar{G}^{2}\right]+\bar{y}^{-3} \bar{m}^{4}\left[k_{8} F+k_{9} \bar{G}\right]\right) .
\end{aligned}
$$

Here the $(\operatorname{det} C)$ has been absorbed into the coefficients $k_{1-9}$. We note that we have no way to determine the renormalisation of $(\operatorname{det} C)$ separately, only that of the coefficients $k_{1-9}$.

We write the divergent contributions to the deformed part of the effective action in the form

$$
\begin{aligned}
\Gamma_{C}^{\text {pole }}= & -\int d^{4} x\left[\bar{y}^{-1}\left(\bar{Z}_{1} F^{3}+\bar{Z}_{2} F^{2} \bar{G}+\bar{Z}_{3} F \bar{G}^{2}+\bar{Z}_{4} \bar{G}^{3}\right)\right. \\
& \left.+\bar{y}^{-2} \bar{m}^{2}\left(\bar{Z}_{5} F^{2}+\bar{Z}_{6} F \bar{G}+\bar{Z}_{7} \bar{G}^{2}\right)+\bar{y}^{-3} \bar{m}^{4}\left(\bar{Z}_{8} F+\bar{Z}_{9} \bar{G}\right)\right] .
\end{aligned}
$$

(Note the overall minus sign, introduced to avoid a proliferation of negative signs later on.) Note also that $\bar{Z}_{1-9}$ will be assumed to contain no finite parts. The divergent diagrams contributing to $\bar{Z}_{1-9}$ can be divided into groups, each group containing diagrams which have the same internal lines and numbers of vertices and also the same number of external $F$ lines; thus only differing in the numbers of external $\bar{\phi}$ lines and attendant $\bar{y}$ or $\bar{m}$ couplings at the vertices. The divergent contributions within each group can be expressed purely in terms of $F$ and $\bar{G}$. In figure 1 are depicted examples of each group at one loop (the one with the maximal number of external $\bar{\phi}$ lines).

Their divergent contributions are shown diagram by diagram in table 1 and given in total by

$$
\begin{aligned}
& \bar{Z}_{1}^{(1)}=\frac{1}{2} k_{2} \frac{L}{\epsilon}, \\
& \bar{Z}_{2}^{(1)}=\left(2 k_{1}+4 k_{2}+3 k_{3}\right) \frac{L}{\epsilon}, \\
& \bar{Z}_{3}^{(1)}=\left(2 k_{2}+4 k_{3}+\frac{5}{2} k_{4}\right) \frac{L}{\epsilon}, \\
& \bar{Z}_{4}^{(1)}=0 \\
& \bar{Z}_{5}^{(1)}=\left(k_{1}+2 k_{2}+k_{3}+k_{6}\right) \frac{L}{\epsilon}, \\
& \bar{Z}_{6}^{(1)}=\left(k_{2}+2 k_{3}+k_{4}+2 k_{5}+4 k_{6}+3 k_{7}\right) \frac{L}{\epsilon}, \\
& \bar{Z}_{7}^{(1)}=0,
\end{aligned}
$$




\begin{tabular}{|c|c|c|c|c|c|c|}
\hline & $\bar{Z}_{1}$ & $\bar{Z}_{2}$ & $\bar{Z}_{3}$ & $\bar{Z}_{5}$ & $\bar{Z}_{6}$ & $\bar{Z}_{8}$ \\
\hline $\mathrm{a}$ & $\frac{1}{2} k_{2}$ & & & & & \\
\hline $\mathrm{b}$ & & $4 k_{2}$ & & $2 k_{2}$ & & \\
\hline $\mathrm{c}$ & & $2 k_{1}$ & & $k_{1}$ & & \\
\hline $\mathrm{d}$ & & $3 k_{3}$ & & $k_{3}+k_{6}$ & & \\
\hline$\overline{\mathrm{e}}$ & & & $4 k_{3}$ & & $2\left(k_{3}+2 k_{6}\right)$ & $2 k_{6}$ \\
\hline$f$ & & & $2 k_{2}$ & & $k_{2}+2 k_{5}$ & $k_{5}$ \\
\hline $\mathrm{g}$ & & & $\frac{5}{2} k_{4}$ & & $k_{4}+3 k_{7}$ & $k_{7}+k_{9}$ \\
\hline
\end{tabular}

Table 1: Divergent contributions from figure 1]

$$
\begin{aligned}
& \bar{Z}_{8}^{(1)}=\left(k_{5}+2 k_{6}+k_{7}+k_{9}\right) \frac{L}{\epsilon}, \\
& \bar{Z}_{9}^{(1)}=0,
\end{aligned}
$$

where

$$
L=\frac{y \bar{y}}{16 \pi^{2}}
$$

(In table 1 the factors of $\frac{L}{\epsilon}$ are suppressed.) These divergences are cancelled as usual by replacing the parameters $y, \bar{y}, k_{1-9}$ and the fields $\phi, \bar{\phi}, F, \bar{F}, \psi, \bar{\psi}$ by corresponding appropriately-chosen bare quantities $y_{B}, \bar{y}_{B}, k_{1 B-9 B}, \phi_{B}, \bar{\phi}_{B}, F_{B}, \bar{F}_{B}, \psi_{B}, \bar{\psi}_{B}$, with the bare fields $\phi_{B}, \bar{\phi}_{B}, \psi_{B}, \bar{\psi}_{B}$, given by $\phi_{B}=Z^{\frac{1}{2}} \phi$, etc. (In the case of the simple WessZumino model, the same $Z$ is used for each bare field.) However, there is a subtlety relating to $F_{B}, \bar{F}_{B}$. In the case of the gauged $\mathcal{N}=1$ and $\mathcal{N}=\frac{1}{2}$ Wess-Zumino models we found it necessary to make non-linear renormalisations of $F, \bar{F}$ in order to ensure multiplicative renormalisability. Here it is not obligatory but nonetheless we shall explore the freedom of making such renormalisations, which will introduce an arbitrariness in the $\beta$-functions for $k_{i}$. Specifically we shall put

$$
\begin{aligned}
F_{B}= & Z^{\frac{1}{2}} F \\
\bar{F}_{B}= & Z^{\frac{1}{2}} \bar{F}+\bar{y}_{B}^{-1}\left(\frac{1}{2} R_{B} F_{B}^{2}+S_{B} F_{B} \bar{G}_{B}+\frac{1}{2} T_{B} \bar{G}_{B}^{2}\right) \\
& +\bar{m}_{B}^{2}\left(U_{B} F_{B}+V_{B} \bar{G}_{B}\right)+\bar{y}_{B} \bar{m}_{B}^{4} W_{B},
\end{aligned}
$$

where $R_{B}, S_{B}, T_{B}, U_{B}, V_{B}$ and $W_{B}$ contain divergent contributions only.

Moreover, the non-renormalisation theorem leads to

$$
y_{B}=\mu^{\frac{1}{2} \epsilon} Z^{-\frac{3}{2}} y, \quad \bar{y}_{B}=\mu^{\frac{1}{2} \epsilon} Z^{-\frac{3}{2}} \bar{y}, \quad m_{B}=Z^{-1} m, \quad \bar{m}_{B}=Z^{-1} \bar{m},
$$

where $\mu$ is the usual dimensional regularisation mass parameter, and hence

$$
\bar{G}_{B}=Z^{-\frac{1}{2}}\left(\mu^{\frac{1}{2} \epsilon} \bar{y} \bar{\phi}^{2}+\bar{m} \bar{\phi}\right) .
$$




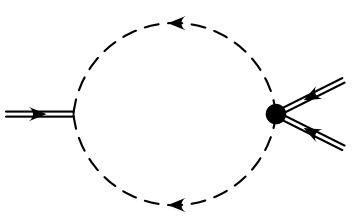

(a)

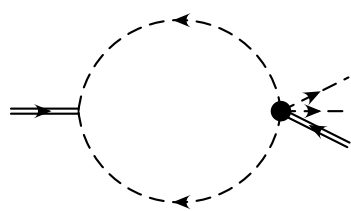

(d)



$(\mathrm{g})$

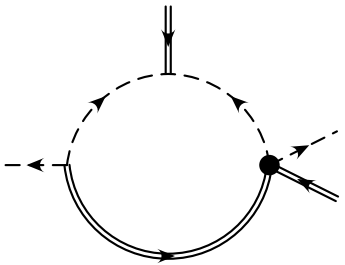

(b)

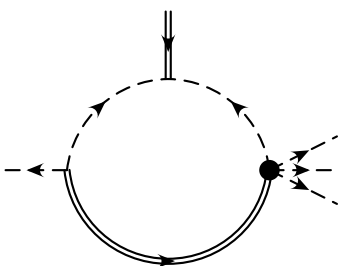

(e)

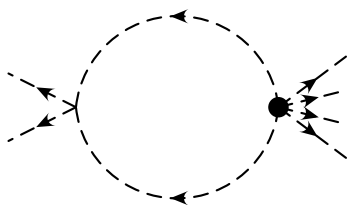

(h)

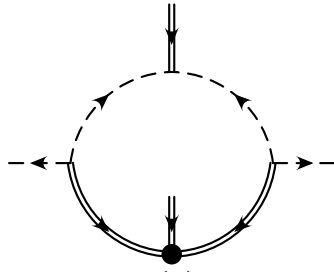

(c)

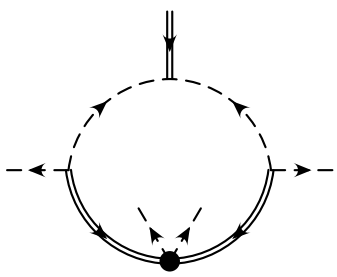

(f)

Figure 1: One-loop Diagrams (dashed, full, double lines representing scalar, fermion and auxiliary fields respectively)

Writing

$$
\begin{aligned}
& k_{i B}=\mu^{\epsilon}\left(k_{i}+\sum_{n=1} k_{i B}^{(n)}\right), \quad i=1 \ldots 4, \\
& k_{i B}=\mu^{\epsilon}\left(k_{i}+\sum_{n=1} k_{i B}^{(n)}\right), \quad i=5,6,7, \\
& k_{i B}=\mu^{\epsilon}\left(k_{i}+\sum_{n} k_{i B}^{(n)}\right), \quad i=8,9,
\end{aligned}
$$

noting that $(\operatorname{det} C)$ has dimension 2 , and where $n$ counts the loop order, and

$$
k_{i B}^{(n)}=\sum_{m=1}^{n} \frac{\kappa_{i}^{(n, m)}}{\epsilon^{m}}, \quad i=1 \ldots 9,
$$


we find (from the bare version of eq. (4.4))

$$
\begin{aligned}
k_{1 B}^{(1)} & =6 \bar{Z}_{1}^{(1)}-3 Z^{(1)} k_{1}-3 R_{B}^{(1)} \\
k_{2 B}^{(1)} & =2 \bar{Z}_{2}^{(1)}-2 Z^{(1)} k_{2}+\left(R_{B}^{(1)}-2 S_{B}^{(1)}\right), \\
k_{3 B}^{(1)} & =2 \bar{Z}_{3}^{(1)}-Z^{(1)} k_{3}+\left(2 S_{B}^{(1)}-T_{B}^{(1)}\right) \\
k_{4 B}^{(1)} & =6 \bar{Z}_{4}^{(1)}+3 T_{B}^{(1)} \\
k_{5 B}^{(1)} & =2 \bar{Z}_{5}^{(1)}-2 Z^{(1)} k_{5}-2 U_{B}^{(1)} \\
k_{6 B}^{(1)} & =\bar{Z}_{6}^{(1)}-Z^{(1)} k_{6}+\left(U_{B}^{(1)}-V_{B}^{(1)}\right) \\
k_{7 B}^{(1)} & =2 \bar{Z}_{7}^{(1)}+2 V_{B}^{(1)} \\
k_{8 B}^{(1)} & =\bar{Z}_{8}^{(1)}-Z^{(1)} k_{8}-W_{B}^{(1)} \\
k_{9 B}^{(1)} & =\bar{Z}_{9}^{(1)}+W_{B}^{(1)} .
\end{aligned}
$$

$\left(R_{B}, S_{B}, T_{B}\right.$ have similar expansions to $k_{1 B-4 B}$ in eqs. (4.11), (4.12), but with, for example,

$$
R_{B}=\sum_{m=1}^{n} \frac{r^{(n, m)}}{\epsilon^{m}} ;
$$

while $U_{B}, V_{B}$ have similar expressions to $k_{5 B-7 B}$ and $W_{B}$ to $k_{8 B, 9 B}$.) We then find (writing $\beta_{i}=\mu \frac{d}{d \mu} k_{i}$ and as usual requiring that $k_{i B}$ in eq. (4.11) be independent of $\mu$ ) that

$$
\beta_{i}^{(1)}=\kappa_{i}^{(1,1)}, \quad i=1 \ldots 9 .
$$

The $\beta$-functions for $y, \bar{y}$ are defined similarly; we have

$$
Z^{(1)}=-\frac{L}{\epsilon}
$$

and then by virtue of eq. (4.9)

$$
\beta_{y}^{(1)}=\frac{3}{2} L y
$$

with a similar expression for $\beta_{\bar{y}}^{(1)}$. From eqs. (4.6), (4.11), (4.16), and writing $r^{(1,1)}=r_{1} L$, etc, we have

$$
\begin{aligned}
& \beta_{1}^{(1)}=\kappa_{1}^{(1,1)}=3\left(k_{1}+k_{2}-r_{1}\right) L, \\
& \beta_{2}^{(1)}=\kappa_{2}^{(1,1)}=\left(4 k_{1}+10 k_{2}+6 k_{3}+r_{1}-2 s_{1}\right) L, \\
& \beta_{3}^{(1)}=\kappa_{3}^{(1,1)}=\left(4 k_{2}+9 k_{3}+5 k_{4}+2 s_{1}-t_{1}\right) L, \\
& \beta_{4}^{(1)}=\kappa_{4}^{(1,1)}=3 t_{1} L, \\
& \beta_{5}^{(1)}=\kappa_{5}^{(1,1)}=2\left(k_{1}+2 k_{2}+k_{3}+k_{5}+k_{6}-u_{1}\right) L, \\
& \beta_{6}^{(1)}=\kappa_{6}^{(1,1)}=\left(k_{2}+2 k_{3}+k_{4}+2 k_{5}+5 k_{6}+3 k_{7}+u_{1}-v_{1}\right) L, \\
& \beta_{7}^{(1)}=\kappa_{7}^{(1,1)}=2 v_{1} L, \\
& \beta_{8}^{(1)}=\kappa_{8}^{(1,1)}=\left(k_{5}+2 k_{6}+k_{7}+k_{8}+k_{9}-w_{1}\right) L, \\
& \beta_{9}^{(1)}=\kappa_{9}^{(1,1)}=w_{1} L .
\end{aligned}
$$


The results adopt their simplest form for $r_{1}=s_{1}=t_{1}=u_{1}=v_{1}=w_{1}=0$, in which case the $\beta$-functions for $k_{4}, k_{7}$ and $k_{9}$ are identically zero, and therefore the corresponding terms could be omitted from the action. This feature will persist at two loops; though it is worth pointing out that $\beta_{4}, \beta_{7}$ and $\beta_{9}$ would acquire contributions in the case of more than one chiral field if we then included the $y F \psi C \psi$ and $\bar{\phi}^{2} \psi C \psi$ interactions. In this simple case, $r_{1}=s_{1}=t_{1}=u_{1}=v_{1}=w_{1}=0$, we also see that once we have a non-zero $k_{1}$, we inevitably generate $k_{2}$ and $k_{5}$ and thence $k_{3}, k_{6}$ and $k_{8}$. We now see clearly why it was in principle necessary to give the $\bar{y}^{-1} F^{3}$ term its own coupling $k_{1}$ rather than $y \bar{y}$, since in general $\beta_{1} \neq \mu \frac{d}{d \mu} y \bar{y}$; and why we had to introduce all the other terms corresponding to $k_{2-9}$ as well. In fact by taking

$$
r_{1}=k_{2}, \quad s_{1}=t_{1}=u_{1}=v_{1}=w_{1}=0,
$$

we can make $\beta_{1}^{(1)}$ consistent with $k_{1}=y \bar{y}$ corresponding to a coefficient of $y$ for the $F^{3}$ term (as in eq. (3.29); but we cannot use the freedom in choosing $r_{1}, s_{1}, t_{1}, u_{1}, v_{1}, w_{1}$ to maintain $k_{2-9}$ all zero.

If we eliminate $F$ and $\bar{F}$ from the action we find

$$
\begin{aligned}
& F=\bar{G}, \\
& \bar{F}=G+\bar{y}^{-1}\left[\frac{1}{2} k_{1} F^{2}+k_{2} F \bar{G}+\frac{1}{2} k_{3} \bar{G}^{2}\right]+\bar{y}^{-2} \bar{m}^{2}\left[k_{5} F+k_{6} \bar{G}\right]+\bar{y}^{-3} \bar{m}^{4} k_{8}
\end{aligned}
$$

and the action becomes

$$
\begin{aligned}
S= & \int d^{4} x\left\{\partial^{\mu} \bar{\phi} \partial_{\mu} \phi+i \bar{\psi} \bar{\sigma}^{\mu} \partial_{\mu} \psi\right. \\
& -G \bar{G}+\frac{1}{2} y \phi \psi^{2}+\frac{1}{2} \bar{y} \bar{\phi}^{2}+\frac{1}{2} m \psi^{2}+\frac{1}{2} \bar{m} \bar{\psi}^{2} \\
& \left.+\frac{1}{6} \lambda_{1} \bar{y}^{-1} \bar{G}^{3}+\frac{1}{2} \bar{m}^{2} \bar{y}^{-2} \lambda_{2} \bar{G}^{2}+\bar{m}^{4} \bar{y}^{-3} \lambda_{3} \bar{G}\right\},
\end{aligned}
$$

where

$$
\begin{aligned}
& \lambda_{1}=k_{1}+3\left(k_{2}+k_{3}\right)+k_{4}, \\
& \lambda_{2}=k_{5}+2 k_{6}+k_{7}, \\
& \lambda_{3}=k_{8}+k_{9} .
\end{aligned}
$$

Writing the divergent contributions to the deformed part of the effective action in the eliminated case as

$$
\Gamma_{C \text { elim }}^{\text {pole }}=-\int d^{4} x\left[Y_{1} \bar{y}^{-1} \bar{G}^{3}+\bar{y}^{-2} \bar{m}^{2} Y_{2} \bar{G}^{2}+\bar{y}^{-3} \bar{m}^{4} Y_{3} \bar{G}\right],
$$

(introducing an overall minus sign as in eq. (4.5)) we have (using minimal subtraction)

$$
\begin{aligned}
& \lambda_{1 B}=\lambda_{1}+6 Y_{1}, \\
& \lambda_{2 B}=\lambda_{2}+2 Y_{2}, \\
& \lambda_{3 B}=\lambda_{3}+Y_{3} .
\end{aligned}
$$


We find from the eliminated diagrams (an example of which is depicted in figure $1(\mathrm{~h})$ ) that

$$
\begin{aligned}
Y_{1}^{(1)} & =\frac{5}{2} \lambda_{1} \frac{L}{\epsilon}, \\
Y_{2}^{(1)} & =\left(\lambda_{1}+3 \lambda_{2}\right) \frac{L}{\epsilon}, \\
Y_{3}^{(1)} & =\left(\lambda_{2}+\lambda_{3}\right) \frac{L}{\epsilon},
\end{aligned}
$$

and then writing

$$
\begin{aligned}
& \lambda_{1 B}=\mu^{\epsilon}\left(\lambda_{1}+\sum_{n=1} \lambda_{1 B}^{(n)}\right), \\
& \lambda_{2 B}=\mu^{\epsilon}\left(\lambda_{2}+\sum_{n=1} \lambda_{2 B}^{(n)}\right), \\
& \lambda_{3 B}=\mu^{\epsilon}\left(\lambda_{3}+\sum_{n} \lambda_{3 B}^{(n)}\right),
\end{aligned}
$$

where

$$
\lambda_{i B}^{(n)}=\sum_{m=1}^{n} \frac{L_{i}^{(n, m)}}{\epsilon^{m}}, \quad i=1 \ldots 3,
$$

we find from eqs. (4.9), (4.10), 4.21), (4.24), (4.25) that

$$
\begin{aligned}
& L_{1}^{(1,1)}=15 \lambda_{1} L \\
& L_{2}^{(1,1)}=\left(2 \lambda_{1}+6 \lambda_{2}\right) L, \\
& L_{3}^{(1,1)}=\left(\lambda_{2}+\lambda_{3}\right) L,
\end{aligned}
$$

and then

$$
\beta_{\lambda_{i}}^{(1)}=L_{i}^{(1,1)}
$$

An important consistency check is that

$$
\begin{aligned}
& \lambda_{1 B}=k_{1 B}+k_{4 B}+3\left(k_{2 B}+k_{3 B}\right), \\
& \lambda_{2 B}=k_{5 B}+2 k_{6 B}+k_{7 B}, \\
& \lambda_{3 B}=k_{8 B}+k_{9 B},
\end{aligned}
$$

and it is easy to confirm that this is satisfied at one loop (irrespective of the values of $R_{B}, S_{B}, T_{B}, U_{B}, V_{B}, W_{B}$ in eq. (4.8)) using eqs. (4.11), (4.12), (4.18), (4.26), (4.27), 4.28). The original deformed Wess-Zumino action of eq. (3.29) corresponded to the values $k_{1}=y \bar{y}$, $k_{2-9}=0$. However, as we emphasised earlier, our more general lagrangian in eq. (4.4) is invariant under $\mathcal{N}=\frac{1}{2}$ transformations whatever the values of $k_{1}-k_{9}$; and we saw from eq. (4.18) that the choice $k_{1}=y \bar{y}, k_{2-9}=0$ is not maintained by renormalisation. It is interesting to ask if there is any complete set of values of $k_{1}-k_{9}$ (or at least any form for 
the deformed action) which is preserved by renormalisation and which would be in some sense natural. To be precise, we ask if we can write

$$
\begin{aligned}
k_{i}=a_{i}(y \bar{y})^{\rho}, & i=1 \ldots 4, \\
k_{i}=a_{i}(y \bar{y})^{\sigma}, & i=5,6,7, \\
k_{i}=a_{i}(y \bar{y})^{\tau}, & i=8,9
\end{aligned}
$$

where $a_{i}, i=1 \ldots 9$ are numbers (i.e. not functions of $y$ or $\bar{y}$, and hence scale independent). This entails

$$
\begin{aligned}
\frac{\beta_{1}^{(1)}}{k_{1}}=\frac{\beta_{2}^{(1)}}{k_{2}}=\frac{\beta_{3}^{(1)}}{k_{3}}=\frac{\beta_{4}^{(1)}}{k_{4}}=\rho\left(\frac{\beta_{y}^{(1)}}{y}+\frac{\beta_{\bar{y}}^{(1)}}{\bar{y}}\right), \\
\frac{\beta_{5}^{(1)}}{k_{5}}=\frac{\beta_{6}^{(1)}}{k_{6}}=\frac{\beta_{7}^{(1)}}{k_{7}}=\sigma\left(\frac{\beta_{y}^{(1)}}{y}+\frac{\beta_{\bar{y}}^{(1)}}{\bar{y}}\right), \\
\frac{\beta_{8}^{(1)}}{k_{8}}=\frac{\beta_{9}^{(1)}}{k_{9}}=\tau\left(\frac{\beta_{y}^{(1)}}{y}+\frac{\beta_{\bar{y}}^{(1)}}{\bar{y}}\right) .
\end{aligned}
$$

Using eqs. (4.17), (4.18), we obtain the equations

$$
\begin{aligned}
(3-3 \rho) k_{1}+3 k_{2}-3 r_{1} & =0, \\
4 k_{1}+(10-3 \rho) k_{2}+6 k_{3}+r_{1}-2 s_{1} & =0, \\
4 k_{2}+(9-3 \rho) k_{3}+5 k_{4}+2 s_{1}-t_{1} & =0, \\
-3 \rho k_{4}+3 t_{1} & =0, \\
k_{2}+2 k_{3}+k_{4}+2 k_{5}+2 k_{3}+(2-3 \sigma) k_{5}+2 k_{6}-2 u_{1} & =0, \\
\left.k_{2}-3 \sigma\right) k_{6}+3 k_{7}+u_{1}-v_{1} & =0, \\
-3 \sigma k_{7}+2 v_{1} & =0, \\
k_{5}+2 k_{6}+k_{7}+(1-3 \tau) k_{8}+k_{9}-w_{1} & =0, \\
-3 \tau k_{9}+w_{1} & =0 .
\end{aligned}
$$

We can solve, for instance, the first three equations in eq. (4.33) successively for $r_{1}$, then $s_{1}$, then $t_{1}$. The fourth then gives the constraint

$$
(15-3 \rho) \lambda_{1}=0
$$

Dealing with the fifth to seventh, and eighth and ninth, equations similarly, we also find

$$
\begin{aligned}
2 \lambda_{1}+(6-3 \sigma) \lambda_{2} & =0 \\
\lambda_{2}+(1-3 \tau) \lambda_{3} & =0 .
\end{aligned}
$$

We then see that these conditions are equivalent to the equations we would have derived using eqs. (4.28), (4.29) if we had sought a similar RG-invariant solution in the eliminated form of the theory. In other words, as was to be expected, the eliminated form of the 
theory contains the same information as the uneliminated form. If, say $\lambda_{1} \neq 0$ (which, through eq. (4.35) implies $\lambda_{2,3} \neq 0$ ) then we require $\rho=5$ but then we can choose $r_{1}, s_{1}$, $t_{1}, u_{1}, v_{1}, w_{1}$ to satisfy eqs. (4.33) for any $k_{i}$; and there are similar solutions with $\lambda_{1}=0$, $\lambda_{2,3} \neq 0, \sigma=2$ and $\lambda_{1}=\lambda_{2}=0, \lambda_{3} \neq 0, \tau=\frac{1}{3}$, all with arbitrary $k_{i}$. In the case

$$
\lambda_{1}=\lambda_{2}=\lambda_{3}=0
$$

(where the deformed potential vanishes in the eliminated case) there is general no constraint on $\rho, \sigma, \tau$; and for any values of $k_{i}$ satisfying eq. (4.36), and any $\rho, \sigma, \tau$, we can again choose $r_{1}, s_{1}, t_{1}, u_{1}, v_{1}, w_{1}$ to satisfy eqs. (4.33). However it is worth pointing out that there are four interesting special solutions with $r_{1}=s_{1}=t_{1}=u_{1}=v_{1}=w_{1}=0$, and with $\rho=\sigma=\tau$, firstly

$$
k_{1}=\frac{1}{4} k_{2}=\frac{3}{8} k_{3}=\frac{1}{2} k_{5}=\frac{3}{4} k_{6}=3 k_{8}, \quad k_{4}=k_{9}=0, \quad \rho=\sigma=\tau=5,
$$

secondly

$$
\begin{aligned}
& k_{1}=k_{2}=-\frac{3}{4} k_{3}, \quad k_{6}=-\frac{5}{3} k_{1}+2 k_{5}, \quad k_{8}=-\frac{2}{3} k_{1}+k_{5}, \\
& k_{4}=k_{7}=k_{9}=0, \quad \rho=\sigma=\tau=2
\end{aligned}
$$

thirdly

$$
k_{1}=-\frac{3}{2} k_{2}=3 k_{3}, \quad k_{5}=-2 k_{6}, \quad k_{4}=k_{7}=k_{9}=0, \quad \rho=\sigma=\tau=\frac{1}{3},
$$

and fourthly

$$
k_{1}=-k_{2}=k_{3}=-k_{4}, \quad k_{5}=-k_{6}=k_{7}, \quad k_{8}=-k_{9}, \quad \rho=\sigma=\tau=0 .
$$

eqs. (4.37)-(4.40) each correspond respectively to one of the general cases mentioned above. eqs. (4.39), (4.40) are particularly intriguing since they will also prove to be valid in a similar way (with no non-linear renormalisation of $\bar{F}$ ) at two loops. This may be related to the fact that eqs. (4.39), (4.40) correspond to actions of the simple form

$$
\begin{aligned}
S= & \int d^{4} x\left\{\partial^{\mu} \bar{\phi} \partial_{\mu} \phi+i \bar{\psi} \bar{\sigma}^{\mu} \partial_{\mu} \psi+\bar{F} F-G F-\overline{G F}\right. \\
& +\frac{1}{2} y \phi \psi^{2}+\frac{1}{2} \bar{y} \bar{\psi}^{2}+\frac{1}{2} m \psi^{2}+\frac{1}{2} \bar{m} \bar{\psi}^{2} \\
& \left.+F\left[\frac{1}{6} k_{1} \bar{y}^{-1}(F-\bar{G})^{2}+\frac{1}{2} k_{5} \bar{y}^{-2} \bar{m}^{2}(F-\bar{G})+k_{8} \bar{y}^{-3} \bar{m}^{4}\right]\right\}
\end{aligned}
$$

or

$$
\begin{aligned}
S= & \int d^{4} x\left\{\partial^{\mu} \bar{\phi} \partial_{\mu} \phi+i \bar{\psi} \bar{\sigma}^{\mu} \partial_{\mu} \psi+\bar{F} F-G F-\overline{G F}\right. \\
& +\frac{1}{2} y \phi \psi^{2}+\frac{1}{2} \bar{y} \overline{\phi \psi}^{2}+\frac{1}{2} m \psi^{2}+\frac{1}{2} \bar{m} \bar{\psi}^{2} \\
& \left.+\frac{1}{6} k_{1} \bar{y}^{-1}(F-\bar{G})^{3}+\frac{1}{2} k_{5} \bar{y}^{-2} \bar{m}^{2}(F-\bar{G})^{2}+k_{8} \bar{y}^{-3} \bar{m}^{4}(F-\bar{G})\right\}
\end{aligned}
$$




\begin{tabular}{|c|c|}
\hline & $\bar{Z}_{1}$ \\
\hline $\mathrm{a}$ & $\frac{1}{2} I k_{2}$ \\
\hline $\mathrm{b}$ & $2 k_{2}$ \\
\hline $\mathrm{c}$ & $k_{1}$ \\
\hline $\mathrm{d}$ & $\frac{3}{2} k_{3}$ \\
\hline
\end{tabular}

Table 2: Divergent contributions from figure 2

respectively. The equations of motion for $F$ and $\bar{F}$ in eq. (4.20) are then particularly simple upon applying eqs. (4.39), 4.40); specifically, that for $F$ becomes linear (in $\bar{G}$ ) upon applying $F=\bar{G}$, the equation for $\bar{F}$. It may also be significant that (again upon applying $F=\bar{G}$ ) these two actions are power-counting super-renormalisable. In this somewhat somewhat restricted sense the values of $k_{1-9}$ in eqs. (4.40), (4.39) may be regarded as the "natural" values we were seeking.

It is also interesting that the values $\rho=2$ and $\rho=\frac{1}{3}$ have a significance even in the massless case, as we see in eqs. (4.38), (4.39), despite these values arising in the first instance for the coefficients of the massive terms in eq. (4.35).

We shall now discuss the two-loop calculation which we shall see follows a very similar pattern. At two loops we have

$$
Z^{(2)}=-I \frac{L^{2}}{\epsilon^{2}}
$$

where

$$
I=1-\frac{1}{2} \epsilon
$$

which leads (through eq. (4.9)) to

$$
\beta_{y}^{(2)}=-\frac{3}{2} L^{2} y
$$

Examples of each group of divergent two-loop diagrams (except for those contributing to $\bar{Z}_{4}, \bar{Z}_{7}$ and $\bar{Z}_{9}$ ) are depicted in figures 246 and the divergent contributions are shown in tables 2 2-⿴囗大 (suppressing factors of $\frac{L^{2}}{\epsilon^{2}}$ ). The diagrams contributing to $\bar{Z}_{4}, \bar{Z}_{7}$ and $\bar{Z}_{9}$ cancel in pairs and in the interests of brevity are not shown explicitly; though in the eliminated case, a similar pair can be seen in figures 0 (a), ](b). This cancellation is due to the fact that for instance the diagrams contributing to $\bar{Z}_{4}$ have a one-loop $\phi^{2} \bar{\phi}^{2}$ subdiagram, and in the uneliminated case there is no counterterm for such a divergence.

The total two-loop divergences are given by

$$
\begin{aligned}
& \bar{Z}_{1}^{(2)}=\frac{L^{2}}{\epsilon^{2}}\left\{k_{1}+\left[\frac{1}{2} I+2\right] k_{2}+\frac{3}{2} k_{3}\right\} \\
& \bar{Z}_{2}^{(2)}=\frac{L^{2}}{\epsilon^{2}}\left\{4[2 I+1] k_{1}+[16 I+15] k_{2}+9[I+2] k_{3}+\frac{15}{2} k_{4}\right\} \\
& \bar{Z}_{3}^{(2)}=\frac{L^{2}}{\epsilon^{2}}\left\{\left[4 k_{1}+20 k_{2}+28 k_{3}+\frac{25}{2} k_{4}\right] I\right\} \\
& \bar{Z}_{4}^{(2)}=0
\end{aligned}
$$




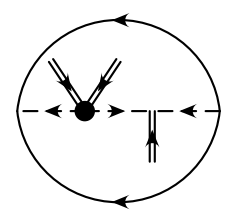

(a)

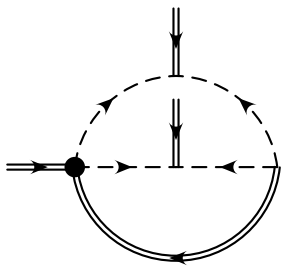

(b)

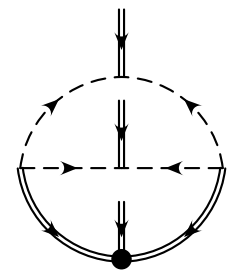

(c)

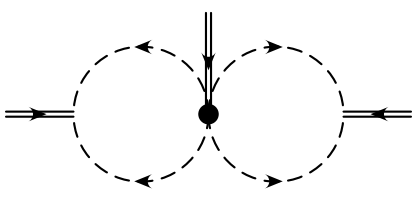

(d)

Figure 2: Two-loop $F^{3}$ Diagrams

$$
\begin{aligned}
\bar{Z}_{5}^{(2)}= & \frac{L^{2}}{\epsilon^{2}}\left\{2\left([2 I+1] k_{1}+\left[4 I+\frac{7}{2}\right] k_{2}+2[I+2] k_{3}+\frac{3}{2} k_{4}\right)\right. \\
& \left.+k_{5}+[I+2] k_{6}+\frac{3}{2} k_{7}\right\}, \\
\bar{Z}_{6}^{(2)}= & \frac{L^{2}}{\epsilon^{2}}\left\{2\left(2 k_{1}+8 k_{2}+10 k_{3}+4 k_{4}\right)+8 k_{5}+16 k_{6}+9 k_{7}\right\} I, \\
\bar{Z}_{7}^{(2)}= & 0 \\
\bar{Z}_{8}^{(2)}= & \frac{L^{2}}{\epsilon^{2}}\left\{k_{1}+3 k_{2}+3 k_{3}+k_{4}+4\left(k_{5}+2 k_{6}+k_{7}\right)+k_{9}\right\} I, \\
\bar{Z}_{9}^{(2)}= & 0 .
\end{aligned}
$$

The two-loop contributions to the bare couplings are given (from the bare form of eq. (4.4) and using eqs. (4.9), (4.10)) by

$$
\begin{aligned}
& k_{1 B}^{(2)}=6 \bar{Z}_{1}^{(2)}-3 Z^{(2)} k_{1}-3\left(Z^{(1)}\right)^{2} k_{1}-3 Z^{(1)}\left(k_{1 B}^{(1)}+3 R_{B}^{(1)}\right)-3 R_{B}^{(2)}, \\
& k_{2 B}^{(2)}=2 \bar{Z}_{2}^{(2)}-2 Z^{(2)} k_{2}-\left(Z^{(1)}\right)^{2} k_{2}-2 Z^{(1)}\left(k_{2 B}^{(1)}-R_{B}^{(1)}+2 S_{B}^{(1)}\right)+R_{B}^{(2)}-2 S_{B}^{(2)}, \\
& k_{3 B}^{(2)}=2 \bar{Z}_{3}^{(2)}-Z^{(2)} k_{3}-Z^{(1)}\left(k_{3 B}^{(1)}-2 S_{B}^{(1)}+T_{B}^{(1)}\right)+2 S_{B}^{(2)}-T_{B}^{(2)}, \\
& k_{4 B}^{(2)}=6 \bar{Z}_{4}^{(2)}+3 T_{B}^{(2)}, \\
& k_{5 B}^{(2)}=2 \bar{Z}_{5}^{(2)}-2 Z^{(2)} k_{5}-\left(Z^{(1)}\right)^{2} k_{5}-2 Z^{(1)}\left(k_{5 B}^{(1)}+2 U_{B}^{(1)}\right)-2 U_{B}^{(2)}, \\
& k_{6 B}^{(2)}=\bar{Z}_{6}^{(2)}-Z^{(2)} k_{6}-Z^{(1)}\left(k_{6 B}^{(1)}-U_{1 B}^{(1)}+V_{B}^{(1)}\right)+U_{B}^{(2)}-V_{B}^{(2)}, \\
& k_{7 B}^{(2)}=2 \bar{Z}_{7}^{(2)}+2 V_{B}^{(2)},
\end{aligned}
$$




\begin{tabular}{|c|cc|}
\hline & $\bar{Z}_{2}$ & $\bar{Z}_{5}$ \\
\hline $\mathrm{a}$ & $12 k_{3}$ & $6 k_{3}$ \\
\hline $\mathrm{b}$ & $6 I k_{3}$ & $3 I k_{3}$ \\
\hline $\mathrm{c}$ & $6 k_{3}$ & $2\left(k_{3}+k_{6}\right)$ \\
\hline $\mathrm{d}$ & $2 I k_{2}$ & $I k_{2}$ \\
\hline $\mathrm{e}$ & $I k_{2}$ & $\frac{1}{2} I k_{2}$ \\
\hline $\mathrm{f}$ & $2 I k_{2}$ & $I k_{2}$ \\
\hline $\mathrm{g}$ & $4 I k_{2}$ & $2 I k_{2}$ \\
\hline $\mathrm{h}$ & $8 k_{2}$ & $4 k_{2}$ \\
\hline $\mathrm{i}$ & $k_{2}$ & $k_{5}$ \\
\hline $\mathrm{j}$ & $2 I k_{2}$ & $I k_{2}$ \\
\hline $\mathrm{k}$ & $3 I k_{3}$ & $I\left(k_{3}+k_{6}\right)$ \\
\hline $\mathrm{l}$ & $2 I k_{2}$ & $I k_{2}$ \\
\hline $\mathrm{m}$ & $\frac{15}{2} k_{4}$ & $\frac{3}{2}\left(2 k_{4}+k_{7}\right)$ \\
\hline $\mathrm{n}$ & $4 k_{1}$ & $2 k_{1}$ \\
\hline $\mathrm{o}$ & $2 I k_{1}$ & $I k_{1}$ \\
\hline $\mathrm{p}$ & $4 I k_{1}$ & $2 I k_{1}$ \\
\hline $\mathrm{q}$ & $2 I k_{1}$ & $I k_{1}$ \\
\hline $\mathrm{r}$ & $4 I k_{2}$ & $2 I k_{2}$ \\
\hline $\mathrm{s}$ & $4 k_{2}$ & $2 k_{2}$ \\
\hline $\mathrm{t}$ & $2 k_{2}$ & $k_{2}$ \\
\hline $\mathrm{u}$ & $-I k_{2}$ & $-\frac{1}{2} I k_{2}$ \\
\hline
\end{tabular}

Table 3: Divergent contributions from figure 3 and 4

$$
\begin{aligned}
& k_{8 B}^{(2)}=\bar{Z}_{8}^{(2)}-Z^{(2)} k_{8}-Z^{(1)}\left(k_{8 B}^{(1)}+W_{B}^{(1)}\right)-W_{B}^{(2)}, \\
& k_{9 B}^{(2)}=\bar{Z}_{9}^{(2)}+W_{B}^{(2)},
\end{aligned}
$$

which yields, using eqs. (4.11), (4.18), (4.43), 4.46),

$$
\begin{aligned}
k_{1 B}^{(2)}= & {\left[3(I+4) k_{1}+3(I+7) k_{2}+9 k_{3}\right] \frac{L^{2}}{\epsilon^{2}}-3 R_{B}^{(2)}, } \\
k_{2 B}^{(2)}= & {\left[16(I+1) k_{1}+(34 I+49) k_{2}+6(3 I+8) k_{3}+15 k_{4}\right] \frac{L^{2}}{\epsilon^{2}}+R_{B}^{(2)}-2 S_{B}^{(2)}, } \\
k_{3 B}^{(2)}= & {\left[8 I k_{1}+4(10 I+1) k_{2}+3(19 I+3) k_{3}+5(5 I+1) k_{4}\right] \frac{L^{2}}{\epsilon^{2}}+2 S_{B}^{(2)}-T_{B}^{(2)}, } \\
k_{4 B}^{(2)}= & 3 T_{B}^{(2)}, \\
k_{5 B}^{(2)}= & {\left[8(I+1) k_{1}+2(8 I+11) k_{2}+4(2 I+5) k_{3}+6 k_{4}+(2 I+5) k_{5}+2(I+4) k_{6}+3 k_{7}\right] \frac{L^{2}}{\epsilon^{2}} } \\
& -2 U_{B}^{(2)},
\end{aligned}
$$




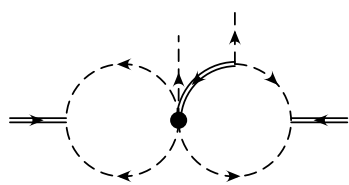

(a)

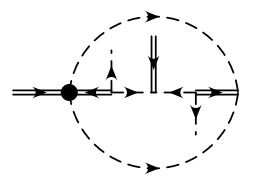

(d)

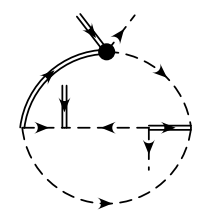

(g)

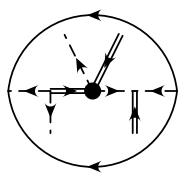

(j)

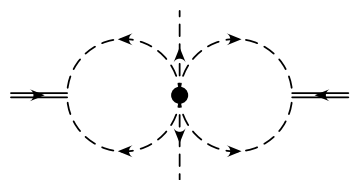

(m)



(b)

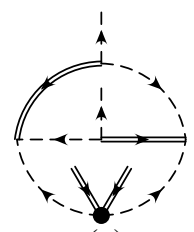

(e)

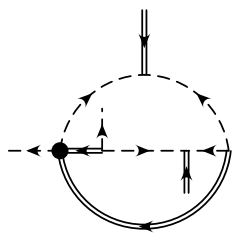

(h)

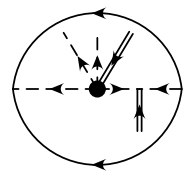

(k)

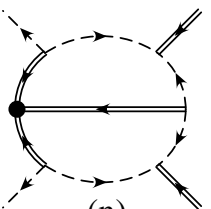

(n)

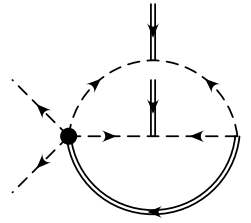

(c)

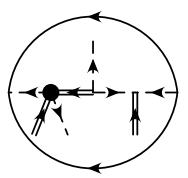

(f)

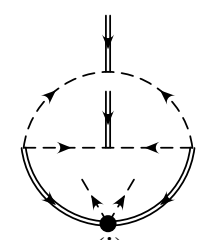

(i)

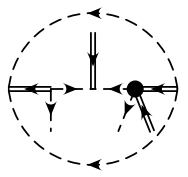

(1)

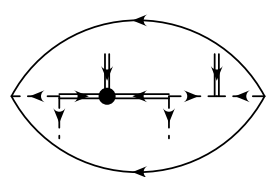

(o)

Figure 3: Two-loop $F^{2} \bar{\phi}^{2}$ Diagrams

$k_{6 B}^{(2)}=\left[4 I k_{1}+(16 I+1) k_{2}+2(10 I+1) k_{3}+(8 I+1) k_{4}+2(4 I+1) k_{5}\right.$ $\left.+(17 I+5) k_{6}+3(3 I+1) k_{7}\right] \frac{L^{2}}{\epsilon^{2}}+U_{B}^{(2)}-V_{B}^{(2)}$,

$k_{7 B}^{(2)}=2 V_{B}^{(2)}$,

$k_{8 B}^{(2)}=\left[I\left(k_{1}+3 k_{2}+3 k_{3}+k_{4}\right)+(4 I+1)\left(k_{5}+2 k_{6}+k_{7}\right)+(I+1)\left(k_{8}+k_{9}\right)\right] \frac{L^{2}}{\epsilon^{2}}-W_{B}^{(2)}$,

$k_{9 B}^{(2)}=W_{B}^{(2)}$.

As at one loop, requiring that $k_{i B}$ as given by eqs. (4.11), (4.12) be $\mu$-independent gives

$$
\beta_{i}^{(2)}=2 \kappa^{(2,1)}
$$

together with the consistency conditions for the two-loop double poles, 




(p)



(s)

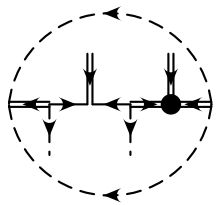

(q)

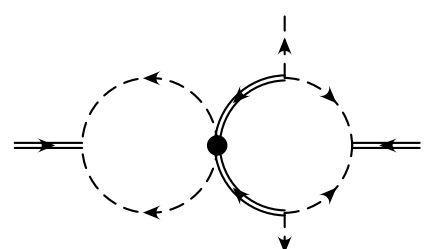

(t)

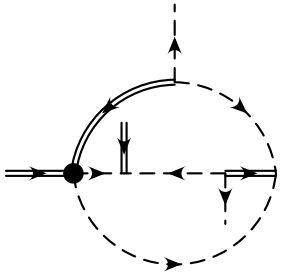

(r)

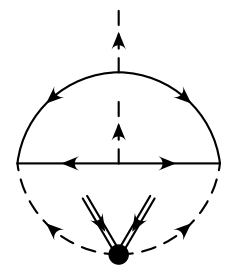

(u)

Figure 4: Two-loop $F^{2} \bar{\phi}^{2}$ Diagrams (continued)

\begin{tabular}{|c|ccc|}
\hline & $\bar{Z}_{3}$ & $\bar{Z}_{6}$ & $\bar{Z}_{8}$ \\
\hline $\mathrm{a}$ & $8 I k_{2}$ & $8 I k_{2}$ & $2 I k_{2}$ \\
\hline $\mathrm{b}$ & $6 I k_{3}$ & $I\left(5 k_{3}+2 k_{6}\right)$ & $I\left(k_{3}+k_{6}\right)$ \\
\hline $\mathrm{c}$ & $2 I k_{3}$ & $I\left(k_{3}+2 k_{6}\right)$ & $I k_{6}$ \\
\hline $\mathrm{d}$ & $2 I k_{3}$ & $I\left(k_{3}+2 k_{6}\right)$ & $I k_{6}$ \\
\hline $\mathrm{e}$ & $2 I k_{3}$ & $I\left(k_{3}+2 k_{6}\right)$ & $I k_{6}$ \\
\hline $\mathrm{f}$ & $4 I k_{1}$ & $4 I k_{1}$ & $I k_{1}$ \\
\hline $\mathrm{g}$ & $2 I k_{2}$ & $I\left(k_{2}+2 k_{5}\right)$ & $I k_{5}$ \\
\hline $\mathrm{h}$ & $4 I k_{2}$ & $2 I\left(k_{2}+2 k_{5}\right)$ & $2 I k_{5}$ \\
\hline $\mathrm{i}$ & $2 I k_{2}$ & $I\left(k_{2}+2 k_{5}\right)$ & $I k_{5}$ \\
\hline $\mathrm{j}$ & $4 I k_{1}$ & $4 I k_{1}$ & $I k_{1}$ \\
\hline $\mathrm{k}$ & $\frac{5}{2} I k_{4}$ & $I\left(k_{4}+3 k_{7}\right)$ & $I\left(k_{9}+k_{7}\right)$ \\
\hline $\mathrm{l}$ & $10 I k_{4}$ & $I\left(7 k_{4}+6 k_{7}\right)$ & $I\left(k_{4}+3 k_{7}\right)$ \\
\hline $\mathrm{m}$ & $-6 I k_{3}$ & $-I\left(5 k_{3}+2 k_{6}\right)$ & $-I\left(k_{3}+k_{6}\right)$ \\
\hline $\mathrm{n}$ & $-4 I k_{1}$ & $-4 I k_{1}$ & $-I k_{1}$ \\
\hline $\mathrm{o}$ & $12 I k_{3}$ & $2 I\left(5 k_{3}+2 k_{6}\right)$ & $2 I\left(k_{3}+k_{6}\right)$ \\
\hline $\mathrm{p}$ & $4 I k_{2}$ & $4 I k_{2}$ & $I k_{2}$ \\
\hline $\mathrm{q}$ & $-8 I k_{2}$ & $-8 I k_{2}$ & $-2 I k_{2}$ \\
\hline $\mathrm{r}$ & $8 I k_{2}$ & $8 I k_{2}$ & $2 I k_{2}$ \\
\hline $\mathrm{s}$ & $4 I k_{3}$ & $2 I\left(k_{3}+2 k_{6}\right)$ & $2 I k_{6}$ \\
\hline $\mathrm{t}$ & $6 I k_{3}$ & $I\left(5 k_{3}+2 k_{6}\right)$ & $I\left(k_{3}+k_{6}\right)$ \\
\hline
\end{tabular}

Table 4: Divergent contributions from figure 0 and 6 


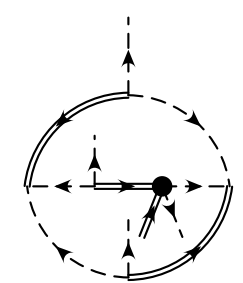

(a)

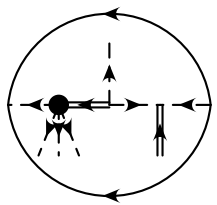

(d)

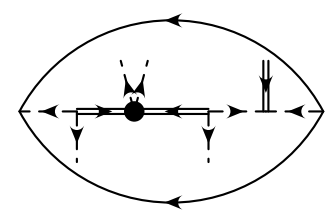

(g)
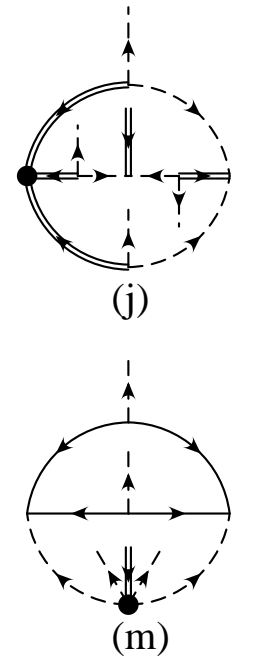

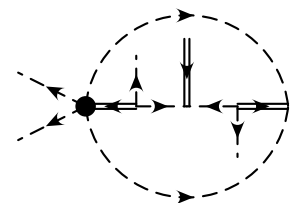

(b)

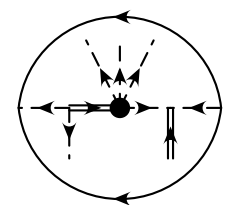

(e)

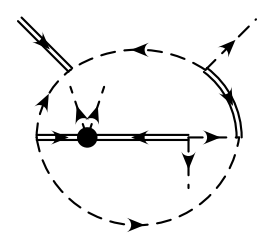

(h)

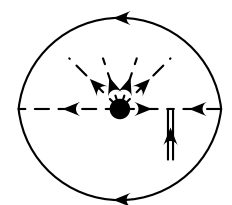

(k)



(n)

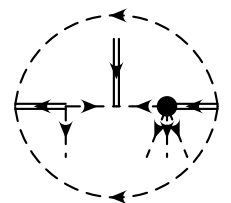

(c)

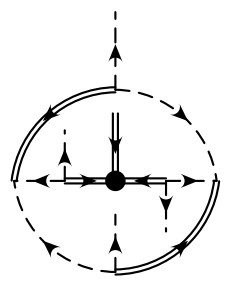

(f)



(i)

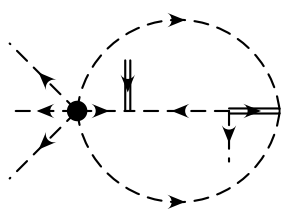

(1)

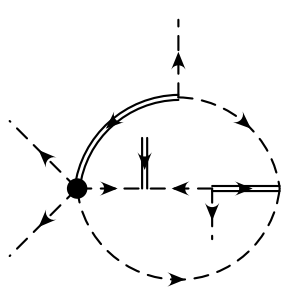

(o)

Figure 5: Two-loop $F \bar{\phi}^{4}$ Diagrams

$$
\begin{aligned}
2\left(16 \pi^{2}\right) \kappa_{1}^{(2,2)}= & 3\left(\kappa_{1}^{(1,1)}+\kappa_{2}^{(1,1)}\right) y \bar{y}+3\left(\beta_{y}^{(1)} \bar{y}+y \beta_{\bar{y}}^{(1)}\right)\left(k_{1}+k_{2}\right), \\
2\left(16 \pi^{2}\right) \kappa_{2}^{(2,2)}= & 2\left(2 \kappa_{1}^{(1,1)}+5 \kappa_{2}^{(1,1)}+3 \kappa_{3}^{(1,1)}\right) y \bar{y} \\
& +2\left(\beta_{y}^{(1)} \bar{y}+y \beta_{\bar{y}}^{(1)}\right)\left(2 k_{1}+5 k_{2}+3 k_{3}\right), \quad \text { etc. }
\end{aligned}
$$




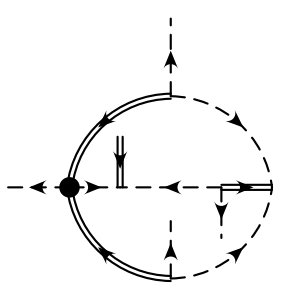

(p)



(s)

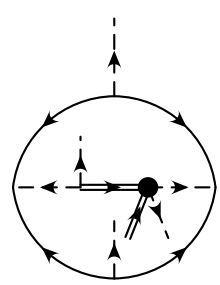

(q)

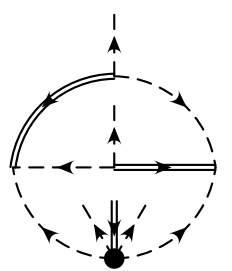

(t)

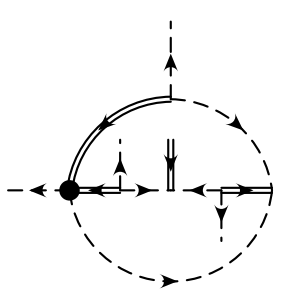

(r)

Figure 6: Two-loop $F \bar{\phi}^{4}$ Diagrams (continued)

\begin{tabular}{|c|ccc|}
\hline & $\bar{G}^{3}$ & $\bar{m}^{2} \bar{G}^{2}$ & $\bar{m}^{4} \bar{G}$ \\
\hline a & $-5 I \lambda_{1}$ & $-\frac{9}{2} I \lambda_{1}-6 I \lambda_{2}$ & $-I\left(\lambda_{1}+5 \lambda_{2}+2 \lambda_{3}\right)$ \\
\hline b & $5 I \lambda_{1}$ & $\frac{9}{2} I \lambda_{1}+6 I \lambda_{2}$ & $I\left(\lambda_{1}+5 \lambda_{2}+2 \lambda_{3}\right)$ \\
\hline c & $10 I \lambda_{1}$ & $7 I \lambda_{1}+6 I \lambda_{2}$ & $I\left(\lambda_{1}+3 \lambda_{2}\right)$ \\
\hline d & $\frac{5}{2} I \lambda_{1}$ & $I \lambda_{1}+3 I \lambda_{2}$ & $I\left(\lambda_{2}+\lambda_{3}\right)$ \\
\hline e & $\frac{5}{2} \lambda_{1}$ & $\lambda_{1}+3 \lambda_{2}$ & $\left(\lambda_{2}+\lambda_{3}\right)$ \\
\hline f & $\frac{15}{2} \lambda_{1}$ & $3 \lambda_{1}+\frac{3}{2} \lambda_{2}$ & 0 \\
\hline
\end{tabular}

Table 5: Divergent contributions from figure 7

From eqs. (4.11), 4.12), (4.48), 4.49), the two-loop $\beta$-functions are hence given by

$$
\begin{aligned}
& \beta_{1}^{(2)}=-3\left(k_{1}+k_{2}+2 r_{2}\right) L^{2}, \\
& \beta_{2}^{(2)}=-2\left(8 k_{1}+17 k_{2}+9 k_{3}-r_{2}+2 s_{2}\right) L^{2}, \\
& \beta_{3}^{(2)}=-\left(8 k_{1}+40 k_{2}+57 k_{3}+25 k_{4}-4 s_{2}+2 t_{2}\right) L^{2}, \\
& \beta_{4}^{(2)}=6 t_{2} L^{2}, \\
& \beta_{5}^{(2)}=-2\left(4\left[k_{1}+2 k_{2}+k_{3}\right]+k_{5}+k_{6}+2 u_{2}\right) L^{2}, \\
& \beta_{6}^{(2)}=-\left(4 k_{1}+16 k_{2}+20 k_{3}+8 k_{4}+8 k_{5}+17 k_{6}+9 k_{7}-2 u_{2}+2 v_{2}\right) L^{2}, \\
& \beta_{7}^{(2)}=4 v_{2} L^{2}, \\
& \beta_{8}^{(2)}=-\left(k_{1}+3 k_{2}+3 k_{3}+k_{4}+4\left[k_{5}+2 k_{6}+k_{7}\right]+k_{8}+k_{9}+2 w_{2}\right) L^{2}, \\
& \beta_{9}^{(2)}=2 w_{2} L^{2},
\end{aligned}
$$

(writing $r^{(2,1)}=r_{2} L^{2}$, etc), and we can check using eqs. (4.11), (4.12), (4.17), 4.18), 4.48), 


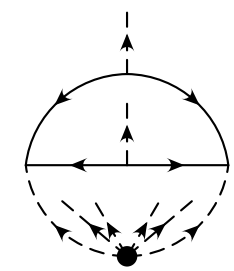

(a)

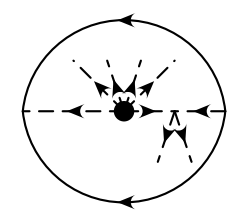

(d)



(b)

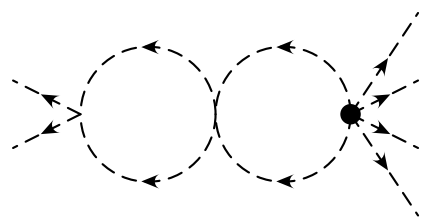

(e)

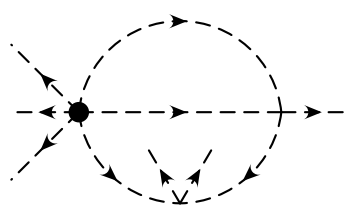

(c)

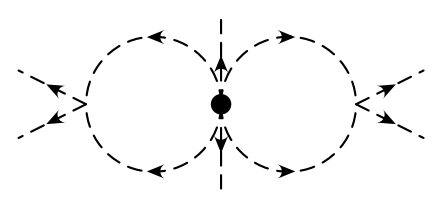

(f)

Figure 7: Two-loop $\bar{\phi}^{6}$ Diagrams in the eliminated case

that eq. 4.50) is satisfied, provided we take

$$
\begin{aligned}
r^{(2,2)} & =\left(r_{1}+s_{1}\right) L^{2}, \\
s^{(2,2)} & =\frac{1}{2}\left(r_{1}+5 s_{1}+3 t_{1}\right) L^{2}, \\
t^{(2,2)} & =0, \\
u^{(2,2)} & =\frac{1}{2}\left(r_{1}+2 s_{1}+t_{1}+u_{1}+v_{1}\right) L^{2}, \\
v^{(2,2)} & =0, \\
w^{(2,2)} & =0 .
\end{aligned}
$$

Of course due to the arbitrariness of the non-linear renormalisations of $\bar{F}$ there is no obvious way of verifying these double pole relations by direct calculation.

Examples of the classes of two-loop diagrams in the eliminated case are depicted in figure 7, and the corresponding divergent contributions are listed in table 5. We find

$$
\begin{aligned}
Y_{1}^{(2)} & =\frac{L^{2}}{\epsilon^{2}}\left[\frac{25}{2} I+10\right] \lambda_{1}, \\
Y_{2}^{(2)} & =\frac{L^{2}}{\epsilon^{2}}[2 I+1]\left[4 \lambda_{1}+\frac{9}{2} \lambda_{2}\right], \\
Y_{3}^{(2)} & =\frac{L^{2}}{\epsilon^{2}}\left\{I \lambda_{1}+[4 I+1] \lambda_{2}+[I+1] \lambda_{3}\right\} .
\end{aligned}
$$

As usual, requiring $\mu$-independence of $\lambda_{i B}$ in eq. (4.26) leads to

$$
\beta_{\lambda_{i}}^{(2)}=2 L_{i}^{(2,1)}
$$


and the consistency conditions for two-loop double poles

$$
\begin{aligned}
& 2\left(16 \pi^{2}\right) L_{1}^{(2,2)}=15\left[L_{1}^{(1,1)} y \bar{y}+\left(\beta_{y}^{(1)} \bar{y}+y \beta_{\bar{y}}^{(1)}\right) \lambda_{1}\right], \\
& 2\left(16 \pi^{2}\right) L_{2}^{(2,2)}=\left(2 L_{1}^{(1,1)}+6 L_{2}^{(1,1)}\right) y \bar{y}+\left(\beta_{y}^{(1)} \bar{y}+y \beta_{\bar{y}}^{(1)}\right)\left(2 \lambda_{1}+6 \lambda_{2}\right), \\
& 2\left(16 \pi^{2}\right) L_{3}^{(2,2)}=\left(L_{2}^{(1,1)}+L_{3}^{(1,1)}\right) y \bar{y}+\left(\beta_{y}^{(1)} \bar{y}+y \beta_{\bar{y}}^{(1)}\right)\left(\lambda_{2}+\lambda_{3}\right) .
\end{aligned}
$$

This leads (via eqs. (4.24), (4.26), (4.27), (4.53)) to $\beta$-functions

$$
\begin{aligned}
& \beta_{\lambda_{1}}^{(2)}=-75 \lambda_{1} L^{2}, \\
& \beta_{\lambda_{2}}^{(2)}=-2\left(8 \lambda_{1}+9 \lambda_{2}\right) L^{2}, \\
& \beta_{\lambda_{3}}^{(2)}=-\left(\lambda_{1}+4 \lambda_{2}+\lambda_{3}\right) L^{2},
\end{aligned}
$$

and we can check using eqs. (4.17), (4.24), (4.26), (4.27), (4.28), (4.53), that eq. (4.55) is satisfied.

As at one loop, eq. (4.30) is crucial for consistency between the uneliminated and eliminated formalisms, and leads to (using eqs. (4.13), (4.24), (4.47))

$$
\begin{aligned}
\bar{Z}_{1}^{(2)}+\bar{Z}_{2}^{(2)}+\bar{Z}_{3}^{(2)}+\bar{Z}_{4}^{(2)}-\frac{1}{2} Z^{(2)}\left(k_{1}+2 k_{2}+k_{3}\right) & \\
-Z^{(1)}\left(3 \bar{Z}_{1}^{(1)}+2 \bar{Z}_{2}^{(1)}+\bar{Z}_{3}^{(1)}\right)+\frac{1}{2}\left(Z^{(1)}\right)^{2}\left(2 k_{1}+3 k_{2}+k_{3}\right) & =\frac{1}{6} \lambda_{1 B}^{(2)}, \\
\bar{Z}_{5}^{(2)}+\bar{Z}_{6}^{(2)}+\bar{Z}_{7}^{(2)}-Z^{(2)}\left(k_{5}+k_{6}\right)-Z^{(1)}\left(2 \bar{Z}_{5}^{(1)}+\bar{Z}_{6}^{(1)}\right) & \\
+\frac{1}{2}\left(Z^{(1)}\right)^{2}\left(3 k_{5}+2 k_{6}\right) & =\frac{1}{2} \lambda_{2 B}^{(2)}, \\
\bar{Z}_{8}^{(2)}+\bar{Z}_{9}^{(2)}-Z^{(2)} k_{8}-Z^{(1)} \bar{Z}_{8}^{(1)}+\left(Z^{(1)}\right)^{2} k_{8} & =\lambda_{3 B}^{(2)} .
\end{aligned}
$$

It is easy to check with the aid of eqs. (4.16), (4.6), (4.43), (4.46), (4.53), that this is satisfied.

As at one loop, if we solve eq. (4.32) we find consistency conditions, in this case

$$
\begin{aligned}
(75+3 \rho) \lambda_{1} & =0 \\
16 \lambda_{1}+(18+3 \sigma) \lambda_{2} & =0, \\
\lambda_{1}+4 \lambda_{2}+(1+3 \tau) \lambda_{3} & =0 .
\end{aligned}
$$

Once again we see that these conditions are equivalent to the equations we would have derived using eqs. (4.56) if we had sought a similar RG-invariant solution in the eliminated form of the theory. Moreover, although as emphasised previously these are the only constraints on $k_{1-9}, \rho, \sigma$ and $\tau$, we see using eq. (4.45) that if we impose either eq. (4.40) or eq. (4.39) then eq. (4.32) is once again satisfied at two loops with $r_{2}=s_{2}=t_{2}=u_{2}=v_{2}=w_{2}=0$. It it intriguing that the values in eqs. (4.40), (4.39) are singled out at both one and two loops.

Finally a few words about the differences between our calculation and that of ref. [17. The authors of ref. 117 identify the coefficient of $(\operatorname{det} C) F^{3}$ with the Yukawa coupling (which they denote as $g$ ), since that is the result of casting the deformed classical superspace 
action into its component form. They also introduce $k_{1}^{\prime} \bar{m}^{4} F$ and $k_{2}^{\prime} \bar{m}^{2} F^{2}$ terms (we have added the prime to distinguish from our own $k_{1}, k_{2}$ which have a different meaning). Therefore, we should be able to read off their results for individual diagrams from tables 14 by writing

$$
\begin{aligned}
k_{1} \rightarrow 2 g \bar{g}(\operatorname{det} C), & k_{5} \rightarrow 8 \bar{g}^{2} k_{2}^{\prime}(\operatorname{det} C), \quad k_{8} \rightarrow 8 \bar{g}^{3} k_{1}^{\prime}(\operatorname{det} C), \\
\epsilon \rightarrow 2 \epsilon, \quad & y \rightarrow 2 g, \quad \bar{y} \rightarrow 2 \bar{g}
\end{aligned}
$$

(including appropriate adjustments for our differing conventions), setting the remaining $k_{i}$ to zero, and remembering the factors of $\bar{y}$ in eq. (4.5). Indeed, our results then agree precisely with those from their eq. (5.10). However, the difference between our results and theirs appears in the two-loop $\beta$-functions. Both we and they eventually derive results in which $F$ and $\bar{G}$ are effectively identified. Taking account of the differences in our definitions, we might expect that our eliminated results would be equivalent to theirs under

$$
\lambda_{1} \rightarrow g \bar{g} \gamma,
$$

where their $\gamma$ is a dimensionless coupling associated with $\operatorname{det} C F^{3}$. However, it is clear that the $\beta$-functions for our $\lambda_{1}$ and their $\gamma$ are in agreement at one loop but not at two loops. Of course it is well-known that $\beta$-functions are scheme-dependent (i.e. dependent on the renormalisation used) beyond one loop in general. The change from one scheme to another may be effected by a redefinition of the couplings of the theory. Nevertheless, we do not believe that the difference betwen our two-loop $\beta$-function and that of ref. [17] is a consequence of using different renormalisation schemes; both we and they are using dimensional regularisation and indeed as we mentioned, our results for individual diagrams agree precisely with theirs, confirming that they correspond to the same renormalisation scheme.

In fact, the difference resides in the precise way of identifying $F$ and $\bar{G}$. In our case this is through eliminating $F$ and $\bar{F}$ via their equations of motion eq. 4.20$)$. One might worry that this equation is only valid at the classical level; but we have shown in detail that we get the same results by eliminating $F$ and $\bar{F}$ at the classical level (hence using the classical version of eq. (4.20)) and then renormalising the eliminated theory; or renormalising the uneliminated theory and then eliminating $F$ and $\bar{F}$ in the renormalised theory (hence using the bare version of eq. (4.20)). This equivalence is expressed by eq. (4.30). There seems little doubt therefore that our procedure is consistent. Moreover there is ample evidence in the literature (at least in the undeformed case) that the same results (for renormalisation constants, $\beta$-functions etc) hold in the eliminated theory obtained by applying the classical equations of motion, as for the uneliminated theory (where calculations are usually performed in superfields of course). The renormalisation eq. (4.8) could be regarded as leading to a quantum modification of the equations of motion for $F$, but we have seen that it has no effect upon the eliminated theory.

On the other hand, the authors of ref. [17] identify $\bar{G}$ and $F$ through a different, and inequivalent, process, which involves reassessing the identification diagrammatically at each loop level. It seems possible that both approaches are internally consistent, but that the eliminated theories thereby obtained are simply different at the quantum level. Both 
approaches lead to consistent two-loop double poles, at least as far as has been computed; it is only the simple poles (and hence the $\beta$-functions) which differ. Let us explain in detail how this happens (and from now on for ease of comparison we shall describe the procedure of ref. 17] using our own notation). Since these authors identify $F$ and $\bar{G}$ immediately, they do not introduce separate couplings for (for instance) $F \bar{G}^{2}, F^{2} \bar{G}, \bar{G}^{3}$ in order to cancel all the different divergent terms; instead they identify all these terms with $F^{3}$ (up to factors) and omit $k_{2-4}$. The subtlety is that they identify $\bar{G}^{2}$ with $\frac{1}{2} F^{2}$ (and presumably $\bar{G}^{3}$ with $\left.\frac{1}{6} F^{3}\right)$. This means that they effectively make the replacement

$$
\begin{aligned}
\bar{Z}_{1} & \rightarrow \bar{Z}_{1}+\bar{Z}_{2}+\frac{1}{2} \bar{Z}_{3}+\frac{1}{6} \bar{Z}_{4}, \\
\bar{Z}_{i} & \rightarrow 0, \quad i=2,3,4, \\
k_{i} & \rightarrow 0, \quad i=2,3,4 .
\end{aligned}
$$

Now in the "eliminated" case, both we and they agree that the double poles in $\lambda_{1 B}^{(2)}$ are as given by eq. (4.55) (equivalent to their eq. (6.5)). On the other hand, since they are starting from the uneliminated calculation, they are deriving $\lambda_{1 B}^{(2)}$ by assuming eq. (4.30), and therefore their double poles in $\lambda_{1 B}^{(2)}$ are given (as are ours) according to eq. (4.57) (but in their case, after applying eq. (4.61)). How can we and they obtain the same double poles despite using different $\bar{Z}_{i}$ in eq. (4.57)? It happens as follows: effectively, through eq. (4.61) they have reduced $Z_{3}^{(2)}$ in eq. (4.57) by $\frac{1}{2}\left(Z_{4}^{(2)}\right.$ is zero in this comparison since we are setting $k_{2-4}=0$ ); and meanwhile the $2 k_{1}$ from $\bar{Z}_{2}^{(1)}$ in eq. (4.6) $\left(\bar{Z}_{3}^{(1)}, \bar{Z}_{4}^{(1)}\right.$ being zero for $\left.k_{2-4}=0\right)$ is effectively transferred to $\bar{Z}_{1}^{(1)}$ which, crucially, has a different factor in eq. (4.57) from $\bar{Z}_{2}^{(1)}$. It turns out that these two alterations have no effect on the double pole in $\lambda_{1 B}^{(2)}$, but change the simple pole and hence the two-loop $\beta$-function for $\lambda_{1}$ (or equivalently $\gamma$ ). However it should be said that their different means of identifying $F$ and $\bar{G}$ at higher loops means that the identification of $\lambda_{1}$ and $\gamma$ is perhaps problematic at higher loops; and there is therefore no clear translation between our results and theirs beyond one loop.

\section{Conclusions}

We have performed a complete analysis up to two loops of the renormalisation of the nonanticommutative Wess-Zumino model. We have shown that in the uneliminated case it is necessary to include all the possible terms which can be generated by renormalisation with their own couplings, and that this leads to results equivalent to those obtained in the eliminated theory. In particular, if one seeks renormalisation-group invariant trajectories for the couplings in the uneliminated theory, one obtains essentially the same solutions as in the eliminated theory; although there are two interesting special solutions for which the uneliminated action adopts a simple form and which require no non-linear renormalisation of the auxiliary fields, at least up to two loops. It would be interesting to see if this behaviour persists to all orders; and also to perform a similar analysis for a gauged model.

In the wider context, some of the earliest investigations of supersymmetry were motivated by the hope that theories might be found which were non-renormalisable by naive 
power counting and yet nevertheless renormalisable in requiring only a finite number of local counter-terms to create a UV-finite effective action. Thanks to the pseudo-symmetries eqs. (4.1), (4.2) the theory studied here (and indeed its generalisation to a gauge theory) provides an explicit realisation of this phenomenon. It is tempting (but presumably fanciful) to speculate on a connection with the recent suggestions [30 that $\mathcal{N}=8$ supergravity (similarly naively non-renormalisable) might in fact be finite. In any event we believe these theories deserve further investigation.

\section{Acknowledgments}

RP was supported by STFC through a graduate studentship. DRTJ was visiting the Aspen Center for Physics and CERN while part of this work was carried out, and gratefully acknowledges financial support from CERN.

\section{References}

[1] R. Casalbuoni, Relativity and Supersymmetries, Phys. Lett. B 62 (1976) 49.

[2] R. Casalbuoni, On the quantization of systems with anticommutating variables, Nuovo Cim. A33 (1976) 115.

[3] L. Brink and J.H. Schwarz, Clifford algebra superspace, CALT-68-813 http://ccdb4fs.kek.jp/cgi-bin/img/allpdf?198102204.

[4] J.H. Schwarz and P. Van Nieuwenhuizen, Speculations concerning A fermionic substructure of space-time, Lett. Nuovo Cim. 34 (1982) 21

[5] S. Ferrara and M.A. Lledó, Some aspects of deformations of supersymmetric field theories, JHEP 05 (2000) 008 hep-th/0002084.

[6] D. Klemm, S. Penati and L. Tamassia, Non(anti)commutative superspace, Class. and Quant. Grav. 20 (2003) 2905 hep-th/0104190.

[7] R. Abbaspur, Generalized noncommutative supersymmetry from a new gauge symmetry, hep-th/0206170.

[8] J. de Boer, P.A. Grassi and P. van Nieuwenhuizen, Non-commutative superspace from string theory, Phys. Lett. B 574 (2003) 98 hep-th/0302078.

[9] H. Ooguri and C. Vafa, The C-deformation of gluino and non-planar diagrams, Adv. Theor. Math. Phys. 7 (2003) 53 hep-th/0302109; Gravity induced C deformation, Adv. Theor. Math. Phys. 7 (2004) 405.

[10] N. Seiberg, Noncommutative superspace, $N=1 / 2$ supersymmetry, field theory and string theory, JHEP 06 (2003) 010 hep-th/0305248.

[11] T. Araki, K. Ito and A. Ohtsuka, Supersymmetric gauge theories on noncommutative superspace, Phys. Lett. B 573 (2003) 209 hep-th/0307076.

[12] R. Britto, B. Feng and S.-J. Rey, Deformed superspace, $N=1 / 2$ supersymmetry and (non) renormalization theorems, JHEP 07 (2003) 067 hep-th/0306215;

Non(anti)commutative superspace, UV/IR mixing and open Wilson lines, JHEP 08 (2003) 001 hep-th/0307091. 
[13] S. Terashima and J.-T. Yee, Comments on noncommutative superspace, JHEP 12 (2003) 053 hep-th/0306237.

[14] R. Britto and B. Feng, $N=1 / 2$ Wess-Zumino model is renormalizable, Phys. Rev. Lett. 91 (2003) 201601 hep-th/0307165.

[15] A. Romagnoni, Renormalizability of $N=1 / 2$ Wess-Zumino model in superspace, JHEP 10 (2003) 016 hep-th/0307209.

[16] O. Lunin and S.-J. Rey, Renormalizability of non(anti)commutative gauge theories with $N=1 / 2$ supersymmetry, JHEP 09 (2003) 045 hep-th/0307275.

[17] M.T. Grisaru, S. Penati and A. Romagnoni, Two loop renormalization for nonanticommutative $N=1 / 2$ supersymmetric WZ model, JHEP 08 (2003) 003 hep-th/0307099.

[18] I. Jack, D.R.T. Jones and L.A. Worthy, One-loop renormalisation of $N=1 / 2$ supersymmetric gauge theory, Phys. Lett. B 611 (2005) 199 hep-th/0412009.

[19] I. Jack, D.R.T. Jones and L.A. Worthy, One-loop renormalisation of general $N=1 / 2$ supersymmetric gauge theory, Phys. Rev. D 72 (2005) 065002 hep-th/0505248.

[20] S. Penati and A. Romagnoni, Covariant quantization of $N=1 / 2 S Y M$ theories and supergauge invariance, JHEP 02 (2005) 064 hep-th/0412041.

[21] M.T. Grisaru, S. Penati and A. Romagnoni, Non(anti)commutative SYM theory: renormalization in superspace, JHEP 02 (2006) 043 hep-th/0510175.

[22] I. Jack, D.R.T. Jones and L.A. Worthy, One-loop renormalisation of $N=1 / 2$ supersymmetric gauge theory with a superpotential, Phys. Rev. D 75 (2007) 045014 hep-th/0701096.

[23] T. Inami and H. Nakajima, Supersymmetric $C P(N) \sigma$-model on noncommutative superspace, Prog. Theor. Phys. 111 (2004) 961 hep-th/0402137.

[24] K. Araki, T. Inami, H. Nakajima and Y. Saito, Quantum corrections in 2D SUSY CP(N-1) $\sigma$-model on noncommutative superspace, JHEP 01 (2006) 109 hep-th/0508061.

[25] B. Chandrasekhar and A. Kumar, $D=2, N=2$, supersymmetric theories on non(anti)commutative superspace, JHEP 03 (2004) 013 hep-th/0310137.

[26] B. Chandrasekhar, $D=2, N=2$ supersymmetric $\sigma$-models on non(anti)commutative superspace, Phys. Rev. D 70 (2004) 125003 hep-th/0408184.

[27] L. Álvarez-Gaumé and M.A. Vazquez-Mozo, On nonanticommutative $N=2 \sigma$-models in two dimensions, JHEP 04 (2005) 007 hep-th/0503016.

[28] B. Chandrasekhar, $N=2 \sigma$-model action on non(anti)commutative superspace, Phys. Lett. B 614 (2005) 207 hep-th/0503116.

[29] I. Jack and R. Purdy, One-loop divergences in the two-dimensional non-anticommutative supersymmetric $\sigma$-model, JHEP 05 (2008) 104 arXiv: 0803.2658].

[30] Z. Bern, J.J. Carrasco, D. Forde, H. Ita and H. Johansson, Unexpected cancellations in gravity theories, Phys. Rev. D 77 (2008) 025010 arXiv:0707.1035. 